\title{
Validating first-principles molecular dynamics calculations of oxide/water interfaces with $x$-ray reflectivity data
}

\author{
Katherine J. Harmon $\odot,{ }^{1, *}$ Kendra Letchworth-Weaver $\odot,{ }^{2,3,4, *}$ Alex P. Gaiduk $\odot,{ }^{3}$ Federico Giberti $\odot,{ }^{3}$ Francois Gygi $\odot,{ }^{5}$ \\ Maria K. Y. Chan $\odot,{ }^{2}$ Paul Fenter $\odot,{ }^{6}$ and Giulia Galli ${ }^{3,7,8, \uparrow}$ \\ ${ }^{1}$ Applied Physics Graduate Program, Northwestern University, Evanston, Illinois 60208, USA \\ ${ }^{2}$ Center for Nanoscale Materials, Argonne National Laboratory, Lemont, Illinois 60439, USA \\ ${ }^{3}$ Pritzker School of Molecular Engineering, University of Chicago, Chicago, Illinois 60637, USA \\ ${ }^{4}$ Department of Physics and Astronomy, James Madison University, Harrisonburg, Virginia, 22807, USA \\ ${ }^{5}$ Department of Computer Science, University of California, Davis, California 95616, USA \\ ${ }^{6}$ Chemical Sciences and Engineering Division, Argonne National Laboratory, Lemont, Illinois 60439, USA \\ ${ }^{7}$ Materials Science Division, Argonne National Laboratory, Lemont, Illinois 60439, USA \\ ${ }^{8}$ Department of Chemistry, University of Chicago, Chicago, Illinois 60637, USA
}

(Received 30 June 2020; revised 16 October 2020; accepted 22 October 2020; published 16 November 2020)

\begin{abstract}
Metal oxide/water interfaces play a crucial role in many electrochemical and photocatalytic processes, such as photoelectrochemical water splitting, the creation of fuel from sunlight, and electrochemical $\mathrm{CO}_{2}$ reduction. First-principles electronic structure calculations can reveal unique insights into these processes, such as the role of the alignment of the oxide electronic energy levels with those of liquid water. An essential prerequisite for the success of such calculations is the ability to predict accurate structural models of these interfaces, which in turn requires careful experimental validation. Here we report a general, quantitative validation protocol for first-principles molecular dynamics simulations of oxide/aqueous interfaces. The approach makes direct comparisons of interfacial x-ray reflectivity (XR) signals from experimental measurements and those obtained from $a b$ initio simulations with semilocal and van der Waals functionals. The protocol is demonstrated here for the case of the $\mathrm{Al}_{2} \mathrm{O}_{3}(001)$ /water interface, one of the simplest oxide/water interfaces. We discuss the technical requirements needed for validation, including the choice of the density functional, the simulation cell size, and the optimal choice of the thermodynamic ensemble. Our results establish a general paradigm for the validation of structural models and interactions at solid/water interfaces derived from first-principles simulations. While there is qualitative agreement between the simulated structures and the experimental best-fit structure, direct comparisons of simulated and measured XR intensities show quantitative discrepancies that derive from both bulk regions (i.e., alumina and water) as well as the interfacial region, highlighting the need for accurate density functionals to properly describe interfacial interactions. Our results show that XR data are sensitive not only to the atomic structure (i.e., the atom locations) but also to the electron-density distributions in both the substrate and at the interface.
\end{abstract}

DOI: 10.1103/PhysRevMaterials.4.113805

\section{INTRODUCTION}

First-principles electronic structure calculations and molecular dynamics (MD) simulations have had a significant impact in materials science, chemistry, and condensed-matter physics [1-7] in the last three decades. However, they rely on several design choices, including theoretical and numerical approximations, whose validation is often a challenging and complex task [7-15]. When performing first-principles

\footnotetext{
*These authors contributed equally to this work.

${ }^{\dagger}$ Author to whom correspondence should be addressed: gagalli@uchicago.edu

Published by the American Physical Society under the terms of the Creative Commons Attribution 4.0 International license. Further distribution of this work must maintain attribution to the author(s) and the published article's title, journal citation, and DOI.
}

calculations, one needs to identify a suitable atomistic model of the system of interest, such as the number of atoms to represent it, and the appropriate thermodynamic conditions (temperature, density, pressure). The level of theory to describe interatomic interactions must be specified, for example, the approximation to density functional theory (i.e., the exchange-correlation functional) that will be used. Upon establishing a theoretical framework, numerical approximations are chosen, e.g., methodological approaches to solve the Kohn-Sham equations. These include the description of core and valence electrons and the basis set to represent the electronic states. Finally, the framework for the calculation of specific properties of interest must be defined. Validation of the results requires that all of these choices and approximations be appropriate for the system of interest, and a tight integration between experiment and computation is essential. To date, most validation procedures are performed for several properties of bulk phases where many direct predictions of experimental observables (e.g., 


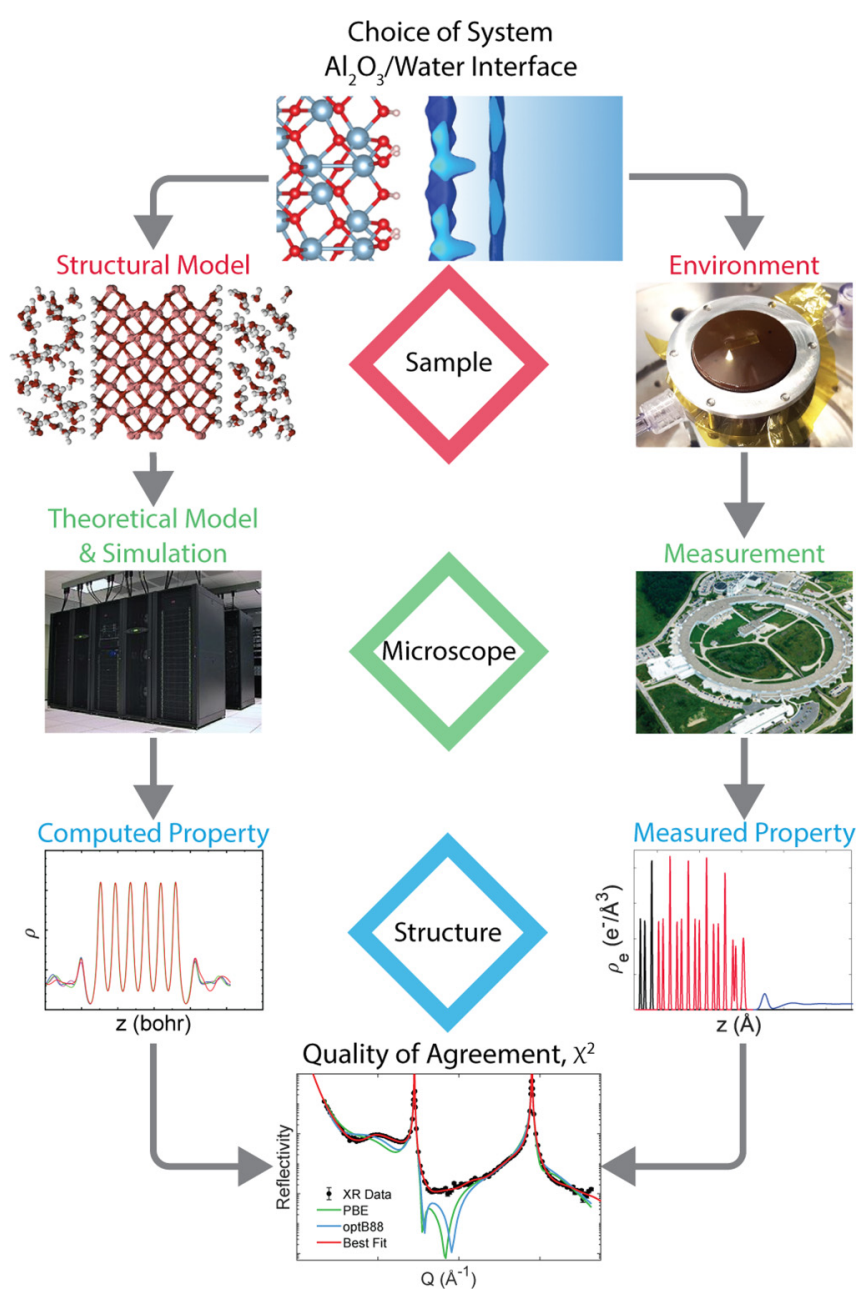

FIG. 1. Schematic of the validation protocol adopted in this work, showing the close coupling of theory and experiment customized for the specific case of first-principles molecular dynamics simulations of the solid/liquid interface using $\mathrm{x}$-ray reflectivity.

crystallographic structures) from theoretical calculations may be obtained $[8,14,16,17]$.

In this work, we focus on the properties of oxide/water interfaces, including their structure (i.e., atom locations) and the associated total electron-density profiles. We present a detailed validation protocol (outlined in Fig. 1) using the metal oxide $\mathrm{Al}_{2} \mathrm{O}_{3}(001) /$ water interface as an example. We compare first-principles molecular dynamics (FPMD) simulation results obtained using semilocal and van der Waals (vdW) functionals and high-resolution specular x-ray reflectivity (XR) measurements, with a focus on defining a general validation protocol. Recent studies have shown that high quality XR data, due to its sensitivity to atomic-scale interfacial structures, provides a strict test of the accuracy of predicted structures from classical MD [18-20] and FPMD [20-22] simulations. Such data are, therefore, promising to build a robust validation tool.

The outline of the paper is as follows. First, we describe the principles of XR in Sec. II and discuss how the reflected intensity can be calculated directly from a simulated interfacial structure (i.e., either from the atom coordinates or the electron-density distributions). We implement the validation protocol in Sec. III A by comparing $a b$ initio predictions of the $\mathrm{Al}_{2} \mathrm{O}_{3}(001) /$ water interfacial structure to experimental XR best-fit results and then evaluate how the choice of theoretical and numerical approximations, including the exchange correlation functional and pseudopotentials (PPs), affects the accuracy of computed XR intensities from the simulations. Next, we explore the effects of the simulation cell size (Sec. III B), of using full DFT-simulated electron distributions to calculate XR signals (Sec. III C), and of sampling multiple independent trajectories (Sec. IIID). We find that significant errors in the description of the aluminum-to-oxygen electronic charge transfer in the $\mathrm{Al}_{2} \mathrm{O}_{3}$ substrate can overwhelm contributions from the interfacial water. In Sec. IIIE we evaluate the accuracy of simulated interfacial water structures, independent of any errors in the simulated substrate, by optimizing the DFT-simulated solid surface structure to minimize its errors with respect to the XR data. We conclude by discussing best practices in theory and simulation necessary to derive robust structural models of aqueous interfaces and discuss work needed to solve remaining challenges in order to achieve full quantitative accuracy (Sec. IV).

\section{METHODS}

\section{A. Specular x-ray reflectivity}

$\mathrm{X}$-ray reflectivity (XR) is a quantitative and sensitive probe of the real-space structure of solid/liquid interfaces [23-25]. The use of hard x rays with photon energies $E>10 \mathrm{keV}$ leads to macroscopic $(>1 \mathrm{~mm})$ penetration lengths through water, enabling the measurement of XR signals from buried solid/liquid interfaces. At these energies, the $\mathrm{x}$-ray wavelength $\lambda<1 \AA$ is comparable to interatomic distances and can, therefore, directly probe the molecular-scale structure of an interface. The reflectivity signal $R(\mathbf{q})$ is the fraction of the incident beam that is reflected from the surface as a function of the scattering vector q. Here we consider the specular, i.e., mirrorlike, x-ray reflectivity where the scattering vector is oriented along the surface normal direction $\hat{z}$ with magnitude $q=\left|\boldsymbol{q}_{z}\right|=(4 \pi / \lambda) \sin (2 \theta / 2) ; 2 \theta$ is the scattering angle between the incident and reflected $\mathrm{x}$ rays as shown in Fig. 2(a). It is convenient also to use the unitless reciprocal lattice vector $L$ to describe the scattering condition such that $q=(2 \pi / c) L$ where $c$ is the bulk unit-cell lattice constant of the solid substrate.

The scattering of $\mathrm{x}$ rays from the bulk crystal lattice planes gives rise to intense Bragg peaks, which reflect the bulk crystalline structure. The experimentally determined lattice constant for $\mathrm{Al}_{2} \mathrm{O}_{3}$ is $c_{\text {exp }}=12.998 \AA$ [26], and its unit cell comprises six lattice planes along the surface normal that differ only in their lateral registry. Consequently, Bragg peaks for $\mathrm{Al}_{2} \mathrm{O}_{3}(001)$ are observed at $L=6 n(n=1,2, \ldots)$ or, equivalently, at $q=n 2.900 \AA^{-1}$ [Fig. 2(b)]. A continuous rod of weak intensity is observed between these Bragg peaks in a form known as a "crystal truncation rod" (CTR) [27]. The $q$-dependent intensity of these weak reflections is directly sensitive to the deviations of the interfacial structure from the expected bulk structure and the associated electron-density profile. Such structural changes include displacements of solid 
(a)

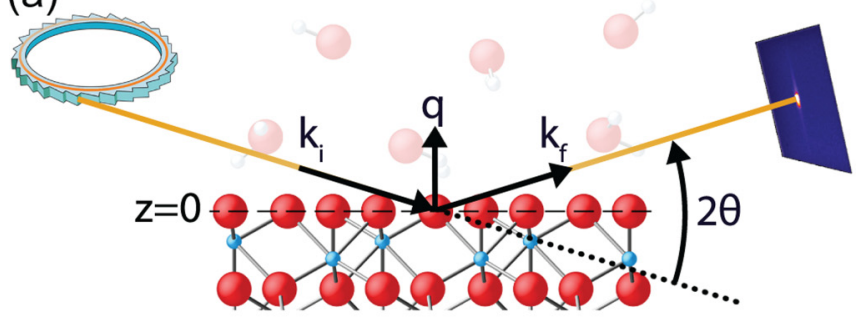

(b)
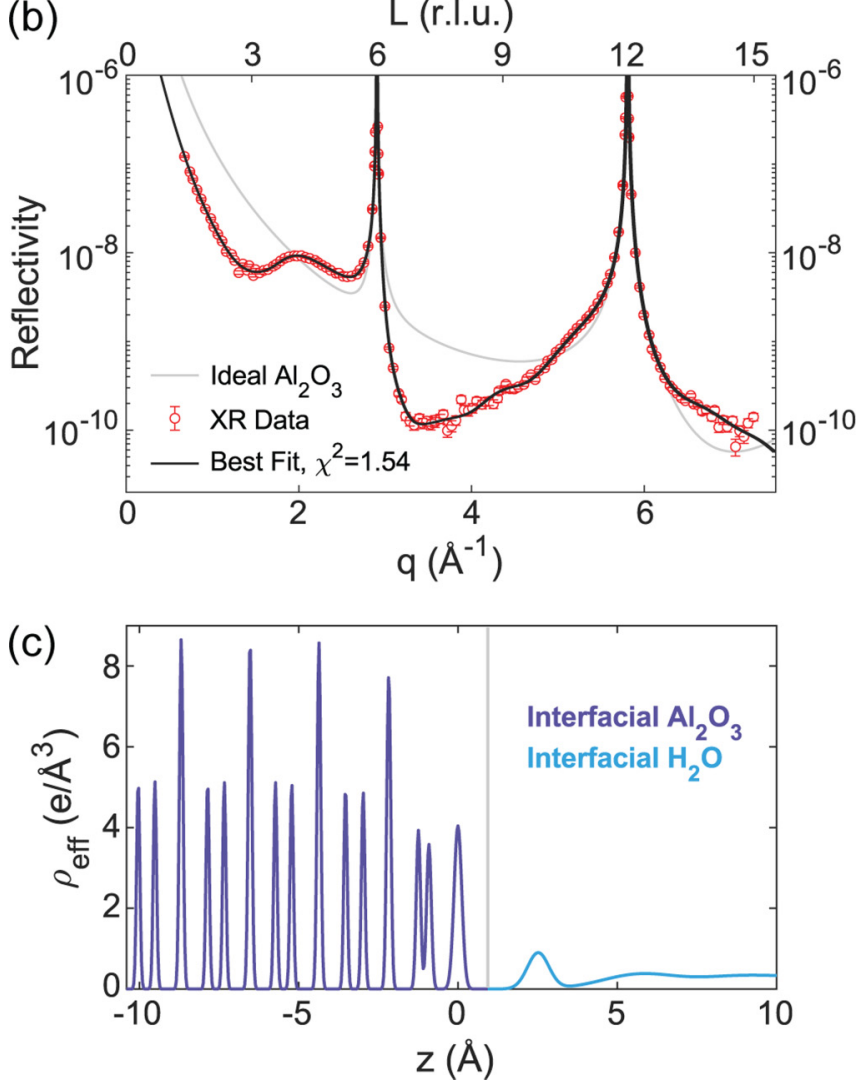

FIG. 2. Experimental schematic and results. (a) Specular x-ray reflectivity from the $\mathrm{Al}_{2} \mathrm{O}_{3}$ /water interface is measured as a function of the vertical momentum transfer $q=\left|k_{\mathrm{f}}-k_{\mathrm{i}}\right|$. (b) The XR data (red circles) are shown as a function of both $q$ (bottom axis) and the reciprocal lattice unit $L$ (r.l.u.) (top axis) with the reflectivity expected from an ideally terminated $\mathrm{Al}_{2} \mathrm{O}_{3}$ substrate (gray line) and the best fit to the data with $\chi^{2}=1.54$ (black line). (c) The best-fit structure of the interface, shown as the effective electron density, is calculated following Eq. (3b). The experimental data and best-fit structure have previously been published in Ref. [21].

surface layers from their bulklike positions resulting from the termination of an infinite crystal and ordering of the liquid near the solid surface [23,24].

The specular XR signal $R(q)$ probes the laterally averaged electron density via the complex structure factor $F(q)$ as

$$
R(q)=T(q)\left(\frac{4 \pi r_{e}}{q A_{U C}}\right)^{2}|F(q)|^{2},
$$

where $T(q)$ describes the $q$-dependent transmission of the $\mathrm{x}$-ray beam through the sample cell, $r_{e}=2.818 \times 10^{-5} \AA$ is the classical electron radius, and $A_{\mathrm{UC}}$ is the unit-cell area of the solid surface. $F(q)$ is the Fourier transform of the electron density $\rho(z)$ [24] as a function of the height $z$ above the surface:

$$
\begin{aligned}
F(q) & =\int_{-\infty}^{\infty} \rho(z) e^{i q z} d z \\
& =\sum_{j=1}^{N_{\text {elements }}} f_{0, j}(q) \int_{-\infty}^{\infty} n_{j}(z) e^{i q z} d z \\
& =\sum_{j=1}^{\infty} f_{0, j}(q) \Theta_{j} e^{-q^{2} u_{j}^{2} / 2} e^{i q z_{j}} .
\end{aligned}
$$

In Eq. (2b), $f_{0, j}$ is the element-specific atomic form factor (FF) [28], $n_{j}(z)$ is the number density profile of each element $j$, and the sum is taken over all $N$ elements in the system (i.e., $\mathrm{Al}, \mathrm{O}$, and $\mathrm{H}$ for the present case of the $\mathrm{Al}_{2} \mathrm{O}_{3} / \mathrm{H}_{2} \mathrm{O}$ interface). Equation (2c) describes the structure factor in terms of the individual atomic layers $j$, where $\Theta_{j}$ is the coverage per substrate unit cell, $z_{j}$ is the height, and $u_{j}$ is the root mean square (rms) width. The Debye-Waller factor, $e^{-q^{2} u_{j}^{2} / 2}$, accounts for thermal fluctuations about the atom locations. Encoded in each FF $f_{0}$ is the spatial distribution of electrons surrounding each atom through the $q$-dependent decay in the scattering strength.

Equation (2) highlights two distinct pathways by which to calculate an XR signal from simulated interfacial structures. The most general approach calculates the XR signals directly from the electron-density profile of the full interface obtained by FPMD electronic structure calculations, thus enabling a comparison between measured and computed XR signals using Eq. (2a). Previous work comparing experimental and FPMD XR signals of the $\mathrm{SrTiO}_{3}$ /water interface used the electron density of the solid surface but did not include the thermal motion of the solid through FPMD and utilized a classical continuum model to describe electron density of the liquid [22]. Another method, which has been reported previously [18-21], calculates the intensities from the simulated elementspecific number density profiles, $n(z)$, or by using parameters to describe $n(z)$ as in Eq. (2b) or Eq. (2c), respectively. This second method mimics the experimental approach that is normally used to analyze XR data through model-dependent fits and has the benefit that it can be computed for any simulated interfacial structure (i.e., from FP or classical MD where the electrons are not explicitly modeled) or proposed interfacial model (as described in Sec. IIB). This approach, however, makes the implicit assumption that atomic FFs (typically for neutral atoms) accurately describe the electron distributions surrounding each atom. This assumption may not be accurate in the presence of interactions that can alter the electrondensity distribution (e.g., covalent bonding, charge transfer, electronic delocalization). As FPMD explicitly determines the charge density distribution around each atom of the system, the results of our calculations allow us to explore the role of different bonding environments at the interface and in bulk regions and thereby assess the strengths and weaknesses of both XR calculation schemes.

The electron density, if not computed directly from FP, can be calculated as the convolution of the elemental number 
density profiles with the inverse Fourier transform of the FFs [Eq. (3a)]. This is distinct from the effective electron-density profile $\rho_{\text {eff }}(z)$, which is the number density profiles weighted by the atomic number $Z_{j}$ of each atomic layer [Eq. (3b)]:

$$
\begin{gathered}
\rho(z)=\sum_{j}\left(\int_{-\infty}^{\infty} f_{0, j}(q) e^{-i q z} d q\right) * n_{j}(z), \\
\rho_{\mathrm{eff}}(z)=\sum_{j} Z_{j} n_{j}(z)=\sum_{j} \frac{Z_{j} \Theta_{j}}{u_{j} \sqrt{2 \pi}} e^{-\left(z-z_{j}\right)^{2} / 2 u_{j}^{2}} .
\end{gathered}
$$

In this work, we evaluate the electron-density distributions in two ways: directly from FPMD simulations and by using FF and number densities as in Eq. (3a). We also present the effective electron-density profile according to Eq. (3b) that can be compared with previous reports on the structure of the $\mathrm{Al}_{2} \mathrm{O}_{3}(001) /$ water interface $[21,29]$.

\section{B. Experiments and data analysis}

The XR measurements, including sample preparation and data analysis, have been described previously [21,29]. Briefly, measurements were carried out using an x-ray photon energy of $12 \mathrm{keV}$. A polished $10 \mathrm{~mm} \times 10 \mathrm{~mm}$ (001)-terminated single-crystal alumina sample was cleaned and mounted in a thin-film cell [24] with ultrapure deionized water (nominal $p \mathrm{H}=7$ ). $\mathrm{XR}$ data [Fig. 2(b)] were analyzed using a model-dependent least-squares fitting procedure. The model describes an effective electron-density profile $\rho_{\text {eff }}(z)$, where each atomic layer is represented as a Gaussian function according to Eq. (3b) with parameters describing its vertical height, coverage, and rms width. The parameters for the bulk $\mathrm{Al}_{2} \mathrm{O}_{3}$ crystal are known [26] and were kept fixed in the data analysis. The parameter values describing the atoms in the near surface region were determined by optimizing the model in an iterative process to achieve a "best-fit" structure in agreement with the XR data. We use a $\chi^{2}$ goodness-of-fit metric for each set of input parameters,

$$
\chi^{2}=\frac{1}{N} \sum_{q_{0}}^{q_{\max }}\left(\frac{R_{\text {meas }}(q)-R_{\text {calc }}(q)}{\sigma(q)}\right)^{2},
$$

where $N$ is the number of data points, and $R_{\text {meas }}, R_{\text {calc }}$, and $\sigma$ are the measured reflectivity, calculated reflectivity from the model, and experimental uncertainty, respectively. $R_{\text {calc }}(q)$ is calculated via Eq. (1) with the model structure factor determined via Eq. (2c) using tabulated atomic FFs $f_{0, j}(q)$ [28]. A perfect fit to the data and its associated uncertainties would result in $\chi^{2}=1$. The optimized model-dependent fit with $\chi^{2}=1.54$ [Fig. 2(b)] gives high confidence in the best-fit structure shown in Fig. 2(c). This interface is described by an oscillatory relaxation pattern within the top five $\mathrm{Al}_{2} \mathrm{O}_{3}$ layers nearest the interface, and the liquid water structure is described by a modulated fluid density with a first adsorbed hydration layer at a distance of $2.52 \pm 0.01 \AA$ above the $\mathrm{Al}_{2} \mathrm{O}_{3}$ surface and an occupation factor of $1.83 \pm 0.12$ water molecules per surface unit cell $\left(\mathrm{H}_{2} \mathrm{O} / \mathrm{UC}\right)$ [21].

\section{Theoretical and numerical methods}

The structural model chosen to represent the $\mathrm{Al}_{2} \mathrm{O}_{3}(001)$ /water interface in our FP simulations consists of a periodically repeated slab where the solid is modeled by $\operatorname{six} \mathrm{Al}_{2} \mathrm{O}_{3}$ layers $\left(\mathrm{Al}-\mathrm{Al}-\mathrm{O}_{3}\right)$ with 12 unit cells in the plane terminated by a fully hydroxylated $\mathrm{Al}_{2} \mathrm{O}_{3}$, and the liquid is modeled with 96 water molecules. The chosen surface termination is consistent with previous experimental characterization and fitting analysis of the $\mathrm{Al}_{2} \mathrm{O}_{3}(001)$ surface in water at neutral $p \mathrm{H}$ [29-34]. The FPMD simulations were carried out with the Qbox code [35] where the Kohn-Sham equations are solved using plane-wave basis sets and optimized norm-conserving Vanderbilt (ONCV) pseudopotentials (PPs) [36,37]. All calculations were performed using PPs where the $1 s$ electrons of oxygen and of aluminum were always considered as core electrons and, thus, frozen in their respective atomic configurations. In the case of $\mathrm{Al}$, we considered two different PPs: (1) a PP in which the $3 s^{2} 3 p^{1}$ electrons were considered as part of the valence partition (the " $3 e^{-} \mathrm{PP}$ ") was used for simulations with two different functionals, PBE [38] and optB88 [39], and (2) a PP in which the $2 s^{2} 2 p^{6} 3 s^{2} 3 p^{1}$ electrons were in the valence partition (the " $11 e^{-} \mathrm{PP}$ ") was used for additional optB88 simulations. We also analyzed the effect of statistical sampling from multiple trajectories and the effect of implicitly including computed electron densities from FP instead of using atomic FF. We compared FPMD results obtained with PBE and optB88, both of which are widely used to study water. The latter explicitly includes van der Waals (vdW) interactions and has previously been shown to outperform PBE in its predictions of the structural and vibrational properties of liquid water [9]. Moreover, optB88 can reproduce some of the water properties as accurately as the hybrid functional PBE0 [40] but at a significantly lower computational cost $[9,41]$.

The initial atomic configuration of the slab used in our FPMD simulations was prepared starting from the final snapshot of a previously equilibrated alumina/water interface, which was simulated using FPMD with the PBE functional and included a three-layer $\mathrm{Al}_{2} \mathrm{O}_{3}$ slab interfaced with 96 water molecules [42]. The snapshot was expanded along the (001) direction to accommodate a thicker six-layer $\mathrm{Al}_{2} \mathrm{O}_{3}$ slab; it was subsequently equilibrated for $\sim 1 \mathrm{~ns}$ using classical MD (CMD) and the LAMMPS code [43] with the ClayFF force field [44] for $\mathrm{Al}_{2} \mathrm{O}_{3}$ and the SPC/E force field [45] to model liquid water. Finally, FPMD was run for $\sim 6$ ps starting from the equilibrated classical simulation. Further details are given in the Supplemental Material [46].

We also carried out CMD calculations to explore the impact of finite size effects on computed XR signals (Table SI and Fig. S1). We tested an alumina model ranging in size from 6 to 24 layers and a liquid model with 280 to 2520 $\mathrm{H}_{2} \mathrm{O}$ molecules and found that while larger simulation may be needed to improve predictive accuracy, our six-layer slab with 96 water molecules in our FP calculations balances the need for accuracy and computational feasibility. In addition, we use CMD to examine in detail the effect of the distance of the first water layer from the solid surface on the XR signals. Further details on the CMD simulations are given in the Supplemental Material [46]. 


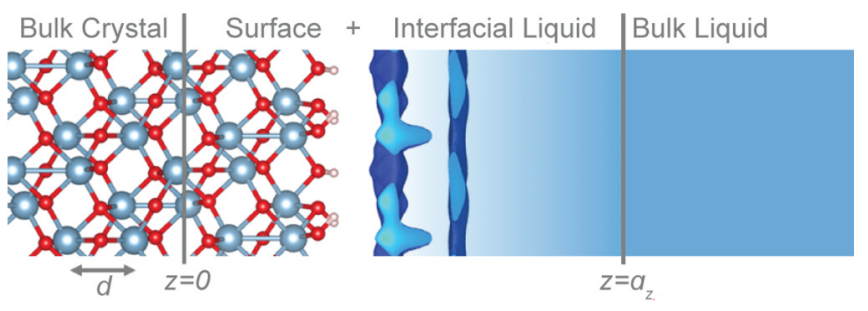

FIG. 3. Definition of bulk crystal, surface, and bulk fluid regions for structure factor calculations. The oxide/water interface region from $0<z<a_{\mathrm{z}}$ represents half of the full FPMD simulation cell from the center of the $\mathrm{Al}_{2} \mathrm{O}_{3}$ slab to the center of the water region, and the bulk regions are semi-infinite extensions thereof. $\mathrm{O}$ and $\mathrm{Al}$ atoms shown as red and slate blue spheres, respectively.

The method used to obtain XR reflectivity from electronic structure calculations using MD trajectories builds on similar calculations carried out for the $\mathrm{SrTiO}_{3} /$ water interface [22]. For each simulation we identify three regions corresponding to the structures probed by the XR measurement: the bulk crystal, the substrate surface and interfacial liquid, and the bulk liquid (Fig. 3). The total structure factor, $F(q)$, is evaluated following Eq. (2a) by writing $\rho(z)$ as the sum of contributions coming from each of the three regions. The contribution of the bulk solid is calculated as a semi-infinite extension of the central (i.e., most bulklike) region of the simulation slab. Specifically, the substrate crystal structure is calculated as a repeating periodic array of the bulklike middle of the $\mathrm{Al}_{2} \mathrm{O}_{3}$ slab (i.e., the three planes $\mathrm{Al}-\mathrm{Al}-\mathrm{O}_{3}$ centered at $z=0$ in Fig. 3) with a slab lattice parameter $d_{006}$ equal to the oxygen-oxygen vertical interlayer spacing; this bulk crystal has a total structure factor given as the product of the structure factor of a single unit cell with density $\rho_{B}(z)$ and the CTR structure factor, $F_{\mathrm{CTR}}=1 /\left(1-e^{-i q d}\right)[24,27]$. The bulk fluid at $z>a_{\mathrm{z}}$ is represented as a semi-infinite water layer above the simulated interfacial water region with uniform density $\rho_{w}$ chosen to be equal to the experimental electron density of bulk water $\left(0.33 e^{-} / \AA^{3}\right)$; its structure factor is $\rho_{w} / i q$ multiplied by a phase factor to describe its distance from the bulk substrate surface, $a_{\mathrm{z}}$. The solid/water interfacial region, $0<z<a_{\mathrm{z}}$, includes the oxide substrate surface and the adjacent fluid with interfacial density, $\rho_{\text {int }}(z)$, as simulated. The full structure factor is then written as

$$
F(q)=\frac{\int_{0}^{d} \rho_{B}(z) e^{i q z} d z}{1-e^{-i q d}}+\int_{0}^{a_{z}} \rho_{\text {int }}(z) e^{i q z} d z+\frac{\rho_{w}}{i q} e^{i q a_{z}} .
$$

The structure factors in Eq. (5) can be calculated directly from the full electronic structure predicted by FPMD, or from the simulated atomic densities and FFs as in previous studies [18,19,21]. The direct use of FPMD electron-density distributions accounts for any changes to valence electron configurations and directly tests the ability of DFT to predict the associated XR signals. Meanwhile, the use of simulated atomic densities is beneficial in evaluating the accuracy of the simulated structure (i.e., the location of a particular atomic layer) with respect to the best-fit structural model and its uncertainties. We employ both calculation schemes in this study to evaluate the consistency between and relative accuracies of the simulated electronic distributions and those assumed by FFs.

Note that when using the slab center to represent the bulk, one faces several issues. The lattice parameter of a finite slab of the size used here does not exactly reproduce the same bulk lattice constant in the middle of the slab that would be obtained from fully periodic bulk calculations [21,47]. Because the XR data are highly sensitive to bulk crystal structures [48], inaccuracies of the slab lattice will introduce significant errors in the computed XR signals (i.e., in the location and intensity of the Bragg peaks). In principle, the slab should contain a bulk region large enough to recover the lattice constant of alumina evaluated with periodic DFT calculations, but the necessary size (possibly several tens of layers), is still outside the current computational capabilities of FPMD on moderate size computers, such as those used in this work. We mitigate errors resulting from the slab lattice parameter by making comparisons with the reflectivity data in units of reciprocal lattice index $L$, which effectively scales the simulated structure to factor out the disagreement in lattice constants.

\section{RESULTS AND DISCUSSION}

\section{A. Choice of theoretical model}

The results of all FPMD simulations discussed in this work, including slab lattice spacings and $\chi^{2}$ levels of agreement with the XR data calculated from elemental number densities and FF or directly from the DFT electron-density distributions, are summarized in Table I. We first discuss the simulations carried out using the PBE functional [Fig. 4(a)]. The simulated atomic number density profile [Fig. 4(b)] obtained from four 10-ps trajectories is qualitatively consistent with the experimentally derived effective electron-density profile [Fig. 2(c)]. A notable, but expected, difference is the appearance of peaks in the simulated profile due to the presence of hydrogen atoms that are not in the experimental density profile. This difference is due to both the weak scattering of $x$ rays from the single hydrogen electron and its delocalization around the proton core.

Despite a satisfactory qualitative agreement, we identify structural discrepancies between PBE and the experimental best-fit model. The PBE slab lattice parameter differed from the experimentally known value by $+1.7 \%$ (Table I). In contrast, the fully periodic, optimized bulk PBE simulation gave an $\mathrm{Al}_{2} \mathrm{O}_{3}$ lattice parameter $1.2 \%$ larger than the experimental value. $\mathrm{PBE}$ also overestimates the interfacial water height above the oxide surface $\Delta_{w}$ by $\sim 0.2 \AA$ compared to the experimental best fit $(2.52 \pm 0.01 \AA)$ and underestimates the occupation factor $\Theta$ of the first hydration layer (i.e., the integrated area of the first water peak with respect to the $\mathrm{Al}_{2} \mathrm{O}_{3}$ surface unit-cell area), giving $1.3 \mathrm{H}_{2} \mathrm{O} / \mathrm{UC}$ compared to $1.83 \pm 0.12 \mathrm{H}_{2} \mathrm{O} / \mathrm{UC}$ found in the experimental analysis. Our PBE results are consistent with those found in previous studies [21,42] and suggest that PBE does not accurately describe the interactions of the $\mathrm{Al}_{2} \mathrm{O}_{3}$ /water interface.

Next, we evaluate how various FPMD design choices affect the structural predictions and directly quantify the accuracy of the simulations in terms of the $\chi^{2}$ level of agreement of computed XR intensities with the experimental XR data. We find that optB88 provides several improvements over PBE 

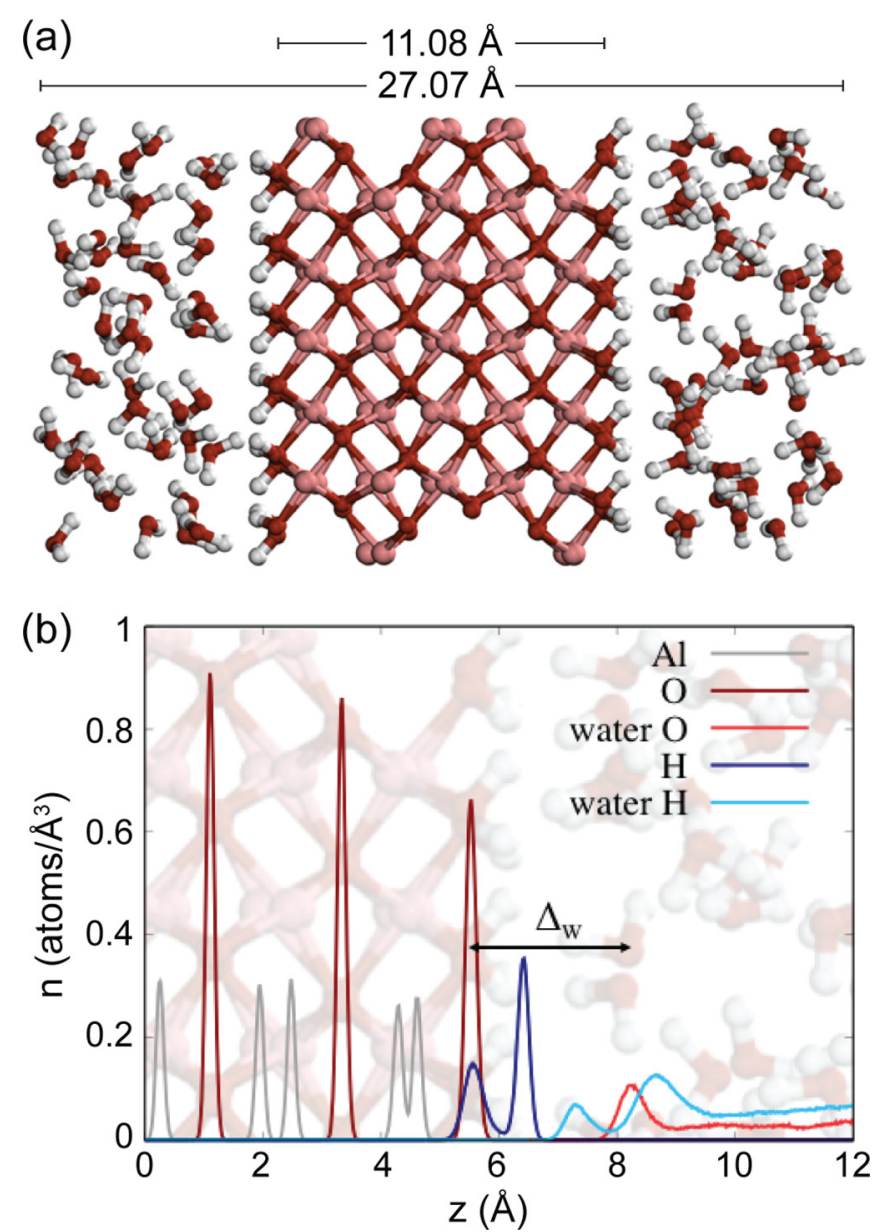

FIG. 4. (a) Snapshot of the initial PBE-based FPMD simulation with a structural model containing a six-layer $\mathrm{Al}_{2} \mathrm{O}_{3}$ slab and 96 water molecules before equilibration. (b) Simulation atomic number density profiles after equilibration. The water height $\Delta_{w}$ is defined by the distance between the surface hydroxyl oxygen and the oxygen of the first layer of water.

with respect to achieving consistency with the experimental best-fit (i.e., atomic) structure. The simulated alumina slab lattice spacing from optB88 exceeds that of the experiment by $+1.5 \%$ as opposed to PBE's $+1.7 \%$ (both using the $3 e^{-}$ $\mathrm{PP})$. Further gains are obtained from optB88 with an $11 e^{-}$ $\mathrm{PP}$, yielding a lattice parameter $+0.8 \%$ compared to that of the experiment (the periodic bulk optB88 DFT calculation gave an $\mathrm{Al}_{2} \mathrm{O}_{3}$ lattice parameter of $2.173 \AA$, $\sim 0.3 \%$ larger than the experimental value). These differences in the $\mathrm{Al}_{2} \mathrm{O}_{3}$ bulk lattice spacing do not lead to substantial qualitative differences between the electron-density profiles of the $\mathrm{Al}_{2} \mathrm{O}_{3}$ surface obtained from PBE, optB88, and the experimental best-fit model [comparisons of the results obtained using the $3 e^{-} \mathrm{PP}$ are shown in Fig. 5(a)]. However, such differences are important in determining the accuracy of the computed XR intensities as all comparisons are performed with respect to $L$ (i.e., after normalizing the lattice constant errors).

We observe clear differences in the interfacial water distributions [Fig. 5(b)]. The optB88 functional predicts an interfacial water height of $2.68 \AA$ (independent of the choice of PP), in closer agreement with the expected experimental value $(2.52 \AA)$ than the height predicted by PBE $(2.74 \AA)$. The optB88 functional also predicts an occupation factor of the first hydration layer of $1.5 \mathrm{H}_{2} \mathrm{O} / \mathrm{UC}$, which is more consistent with the experimental best-fit result $\left(1.83 \pm 0.12 \mathrm{H}_{2} \mathrm{O} / \mathrm{UC}\right)$ than the value determined using PBE $\left(1.3 \mathrm{H}_{2} \mathrm{O} / \mathrm{UC}\right)$. These results suggest that the optB88 functional, which includes $\mathrm{vdW}$ interactions, provides an improved description of interfacial water properties relative to PBE.

The noted improvements in the electron-density distribution obtained with the optB88 functional and $3 e^{-}$PP translate to superior $\chi^{2}$ agreements in the calculation of the optB88 $3 e^{-}$ PP XR intensities [Figs. 5(c) and 5(d) and Table I]. Namely, $\chi^{2}$ decreases from 102 for PBE to 66 for optB88 when using atomic FFs, or from 119 to 91 for PBE and optB88, respectively, when using DFT electron densities. However, such an improvement is not observed when using optB88 with the $11 e^{-}$PP. In this case, $\chi^{2}=100$ using FFs, equivalent to the PBE result (102), and $\chi^{2}=197$ using DFT charge densities. We explore the effects of PP choice on the predicted XR signal in Sec. III C.

Our results highlight two important findings. First, the values of $\chi^{2}$ obtained here are significantly larger than

TABLE I. $\quad \chi^{2}$ from comparing first-principles molecular dynamics (FPMD) simulations and experiment. PBE [38] and optB88 [39] indicate the energy functionals used in the simulations. The pseudopotential PP includes either 3 or 11 valence electrons chosen for Al (see text). Also noted are the volume change $\Delta V$ of the simulation cell via compression along alumina (001) direction relative to the volume used in the PBE simulation; the total simulation time $t$ after averaging over four independent trajectories; the $\mathrm{Al}_{2} \mathrm{O}_{3}$ lattice parameter $d_{006}$; the height $\Delta_{w}$ of the first hydration layer above the $\mathrm{Al}_{2} \mathrm{O}_{3}$ surface; and the water occupation $\Theta$ in the first hydration layer per $\mathrm{Al}_{2} \mathrm{O}_{3}$ surface unit cell (UC). $\chi^{2}$ is calculated both using atomic form factors (FFs) with the FPMD atomic structure prediction and from the DFT electronic structure directly.

\begin{tabular}{|c|c|c|c|c|c|c|c|c|}
\hline \multirow[b]{2}{*}{ Exchange correlation } & \multirow[b]{2}{*}{ PP } & \multirow[b]{2}{*}{$\Delta V(\%)$} & \multirow[b]{2}{*}{$t(\mathrm{ps})$} & \multirow[b]{2}{*}{$d_{006}(\AA)$} & \multirow[b]{2}{*}{$\Delta_{w}(\AA)$} & \multirow[b]{2}{*}{$\Theta\left(\mathrm{H}_{2} \mathrm{O} / \mathrm{UC}\right)$} & \multicolumn{2}{|c|}{$\chi^{2}$} \\
\hline & & & & & & & Atomic FFs & $\mathrm{DFT} e^{-}$ \\
\hline PBE & 3 & 0 & 40 & 2.2028 & 2.74 & 1.3 & 102 & 119 \\
\hline \multirow[t]{4}{*}{ optB88 } & 3 & 0 & 12.8 & 2.1982 & 2.68 & 1.5 & 66 & 91 \\
\hline & 11 & 0 & 11.6 & 2.1843 & 2.68 & 1.5 & 100 & 197 \\
\hline & 11 & 10 & 13.2 & 2.1809 & 2.64 & 1.7 & 69 & 133 \\
\hline & 3 & 10 & & & & & & $57^{\mathrm{a}}$ \\
\hline Experiment & & & & 2.1663 & 2.52 & 1.83 & 1.54 & \\
\hline
\end{tabular}

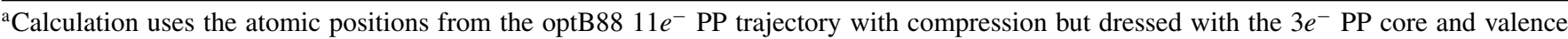
configurations. 

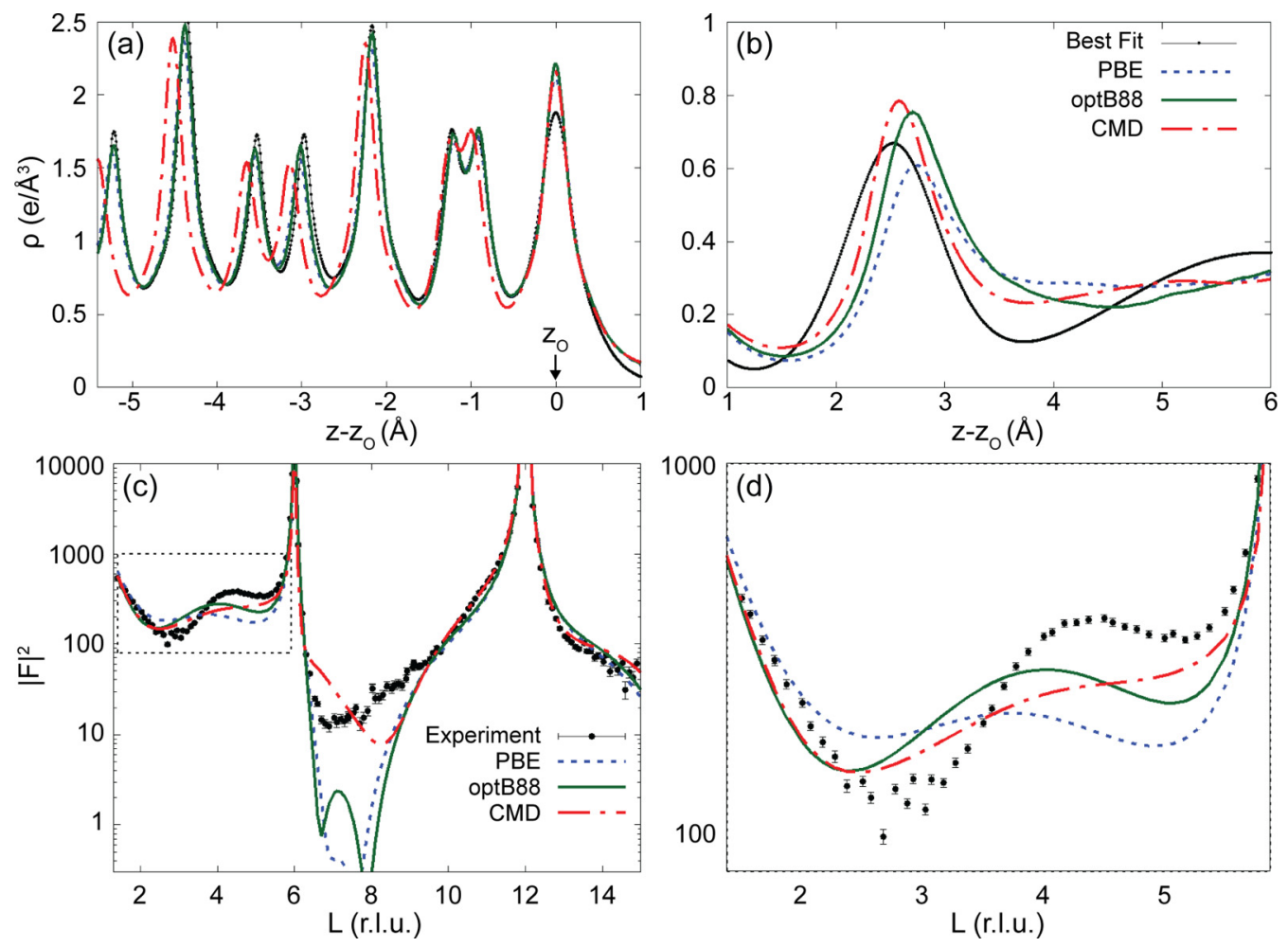

FIG. 5. Comparison between theoretical methods and the experimental result for the electron density $\rho$ and reflected intensity $|F|^{2}$ as a function of reciprocal lattice unit $L$ computed using form factors (FFs). (a) Electron-density profiles of the simulated $\mathrm{Al}_{2} \mathrm{O}_{3}$ surface from first-principles molecular dynamics (FPMD) simulations using the PBE (blue) and optB88 (green) functionals with a $3 e^{-}$pseudopotential (see text), plotted with the surface oxygen at $z_{\mathrm{O}}$ as the reference plane, show subtle deviations from the experimental best fit (black); the differences are more visible in an equivalent classical MD (CMD) simulation (red). (b) The FPMD electron-density profiles of the interfacial water region reveal predicted interfacial water heights exceeding that obtained from XR experiments. (c) The simulations reproduce the general shape of the XR data (experimental errors shown as $1 \sigma$ uncertainties) but with notable differences in the region below the first Bragg peak (dotted box) and for $L \sim 6-9$. (d) A close-up view of the region in the dotted box from (c) shows that the simulated XR intensities from FP qualitatively reproduce oscillations in the XR data better than that from $\mathrm{CMD}$; the optB88 functional outperforms PBE, reflecting important qualitative improvements in the optB88 electron-density distribution.

those obtained through the experimental analysis $\left[\chi^{2}=1.54\right.$; Figs. 2(b) and 2(c)], indicating that the accuracy of the simulations is not yet fully quantitative. Second, the $\chi^{2}$ values are better when using FF than when using the DFT charge densities, revealing that the comparison with experiments is sensitive to both the atomic and electron-density distributions. We explore the sensitivity to the DFT electron distributions in greater detail in Sec. III C.

Examining qualitative features of the computed XR signals [Figs. 5(c) and 5(d)] points to differences in the real-space structures that can guide the validation process. Here, we compare the FPMD results with a CMD calculation $\left(\chi^{2}=55\right.$, Table SI in the Supplemental Material [46]) to gain critical insights. The FPMD XR signals exhibit an order of magnitude deviation from the XR data at $L \sim 6-9$ [Fig. 5(c)] and factors of $\sim 2$ discrepancies near $L=2-5$ [Fig. 5(d)]. However, the CMD XR signal does not show a similar low-intensity feature at $L \sim 6-9$ and is more qualitatively consistent with the experimental data at these scattering conditions. Notably, the CMD interfacial water height of $2.55 \AA$ [Fig. 5(b)] is nearly equivalent to the value obtained from the experimental bestfit model. When the CMD-simulated water layer is rigidly shifted away from the solid $\mathrm{Al}_{2} \mathrm{O}_{3}$ surface and brought in line with the FPMD values, the CMD XR intensity begins to qualitatively reproduce the low-intensity region observed in the FPMD intensities (Fig. S2 of the Supplemental Material [46]). These results suggest that an accurate reproduction of structural details in the interfacial water structure (especially the interfacial water height), is critical but not sufficient to obtain a high-level agreement with the measured data.

\section{B. Volume of the simulation supercell}

In Sec. III A we found that the simulated interfacial water heights $\Delta_{w}$ exceeded that indicated by the experiment and showed that this feature plays an important role in reproducing the XR data. We also found that the simulated adsorbed water densities (i.e., occupation factors) were lower than the experimental result, indicating that the volume chosen in our NVT simulations $\left(3117 \AA^{3}\right)$ may have been too large. In principle, one may determine the optimal slab volume by using NPT simulations. However, adopting such a protocol is currently prohibitive from a computational standpoint. Therefore, we explored the sensitivity of the simulated interfacial water density profile to the selection of simulation volume by reducing the supercell $z$-dimension size by $10 \%$ using simulations performed at the optB88 level. 

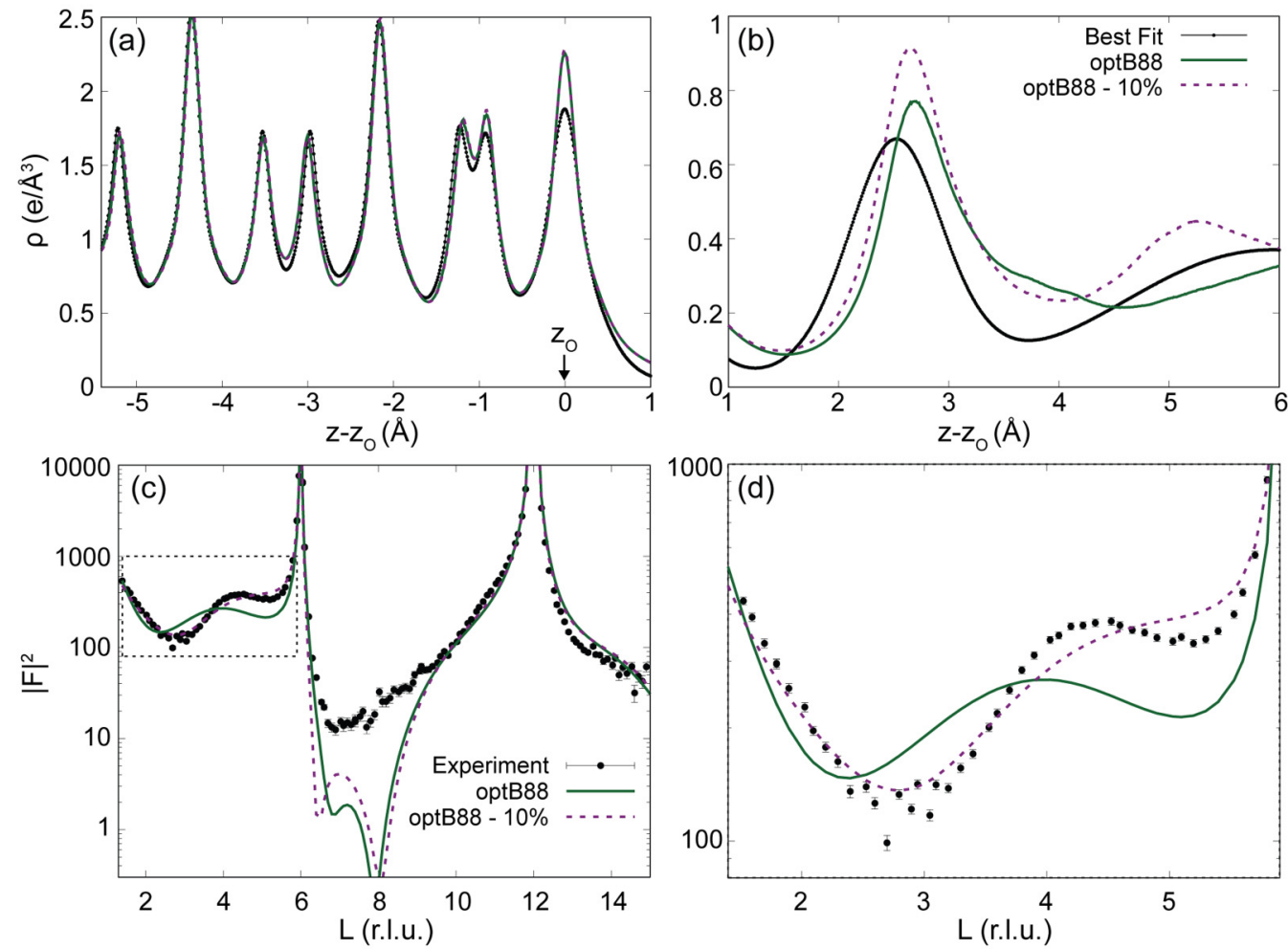

FIG. 6. Effect of a $10 \%$ size reduction of the unit cell along the $\mathrm{Al}_{2} \mathrm{O}_{3}$ surface normal for the case of FPMD simulations with the optB88 functional and $11 e^{-} \mathrm{PP}$ computed using atomic FFs. (a) The electron-density profiles $\rho(\boldsymbol{z})$ of the $\mathrm{Al}_{2} \mathrm{O}_{3}$ interface show equivalent qualitative agreement with the experimental best-fit structure (black) before compression (green) and after compression (purple); the structures are given with the alumina surface oxygen layer $z_{\mathrm{O}}$ as the reference plane. (b) The interfacial water densities increase with volume reduction, and the interfacial water height is in closer agreement with the experimental result. (c) The simulated XR intensities $|F|^{2}$ vs reciprocal lattice unit $L$ (r.l.u.) are generally similar before and after decreasing the simulation cell size but with notable changes in the oscillations below the first Bragg peak (dotted box), as highlighted in (d), bringing the simulated XR signal in closer qualitative agreement with the XR data (experimental errors are shown as $1 \sigma$ uncertainties).

The volume compression in our optB88 simulations (which we refer to as "optB88-10\%") led to an interfacial water density in better agreement with the experimental XR best-fit result. Due to the low compressibility of $\mathrm{Al}_{2} \mathrm{O}_{3}$, the $10 \%$ volume reduction of the supercell did not significantly alter the alumina slab electron-density profile or lattice spacing [Fig. 6(a) and Table I]. We obtained a $\sim 17 \%$ decrease in the volume of the much more compressible water region. The resultant interfacial water profile [Fig. 6(b)] had an integrated first hydration layer density of $1.7 \mathrm{H}_{2} \mathrm{O} / \mathrm{UC}$ and an interfacial water height $0.04 \AA$ closer to the $\mathrm{Al}_{2} \mathrm{O}_{3}$ surface at $2.64 \AA$. We also observe significant changes to the second hydration layer, including an increased density and a peak shift from $>6$ to $5.25 \AA$. Finally, the asymptotic bulklike water density (i.e., the density at the point farthest from the solid surface) was $0.29 e^{-} / \AA^{3}$, in closer agreement with the experimental value of $0.33 e^{-} / \AA^{3}$ than the result from the optB88 simulation using a larger volume $\left(0.22 e^{-} / \AA^{3}\right)$.

The computed XR intensities from the optB88-10\% simulation with the $11 e^{-}$PP [Fig. 6(c)] are overall in substantially better agreement with the XR data than those from the initial optB88 $11 e^{-}$PP calculation (Table I), resulting in $\chi^{2}=$ 69 when using atomic FFs and $\chi^{2}=133$ when using the electron-density prediction directly from DFT. The majority of the $\chi^{2}$ improvement appears to originate from a substantial improvement in the oscillations in the simulated XR signals below the Bragg peak [Fig. 6(d)], indicating that these low- $L$ $\mathrm{XR}$ intensities, in addition to those at $6<L<9$, provide a sensitive fingerprint of the interfacial water structure.

\section{Sensitivity to DFT-simulated electron densities}

We showed that the XR data are sensitive not only to the atomic positions, but also to the electron distribution surrounding each atom. To isolate the impact of the charge densities, we compare three calculations using a single prediction of the atomic density distribution (that of the $11 e^{-}$ PP optB88-10\% simulation) but with different descriptions of the electron configuration: atomic $\mathrm{FF}, 3 e^{-} \mathrm{PP}$, and $11 e^{-}$ PP. These calculations all give similar electron-density distributions [Figs. 7(a)-7(c)]. However, the $11 e^{-}$PP simulation shows a distinct broadening of the electron density within the $\mathrm{Al}_{2} \mathrm{O}_{3}$ slab [Fig. 7(b)]. This apparent delocalization leads to a visible overestimate of the electron density between atomic layers and an associated reduction in the density at the $\mathrm{Al}$ peak positions. We observe a nearly identical delocalization of the charge when carrying out calculations for the same snapshot using either PBE or the hybrid functional PBE0 [40] with the $11 e^{-}$PP (Fig. S3 of the Supplemental Material [46]), indicating that the inaccuracy in the description of charge 

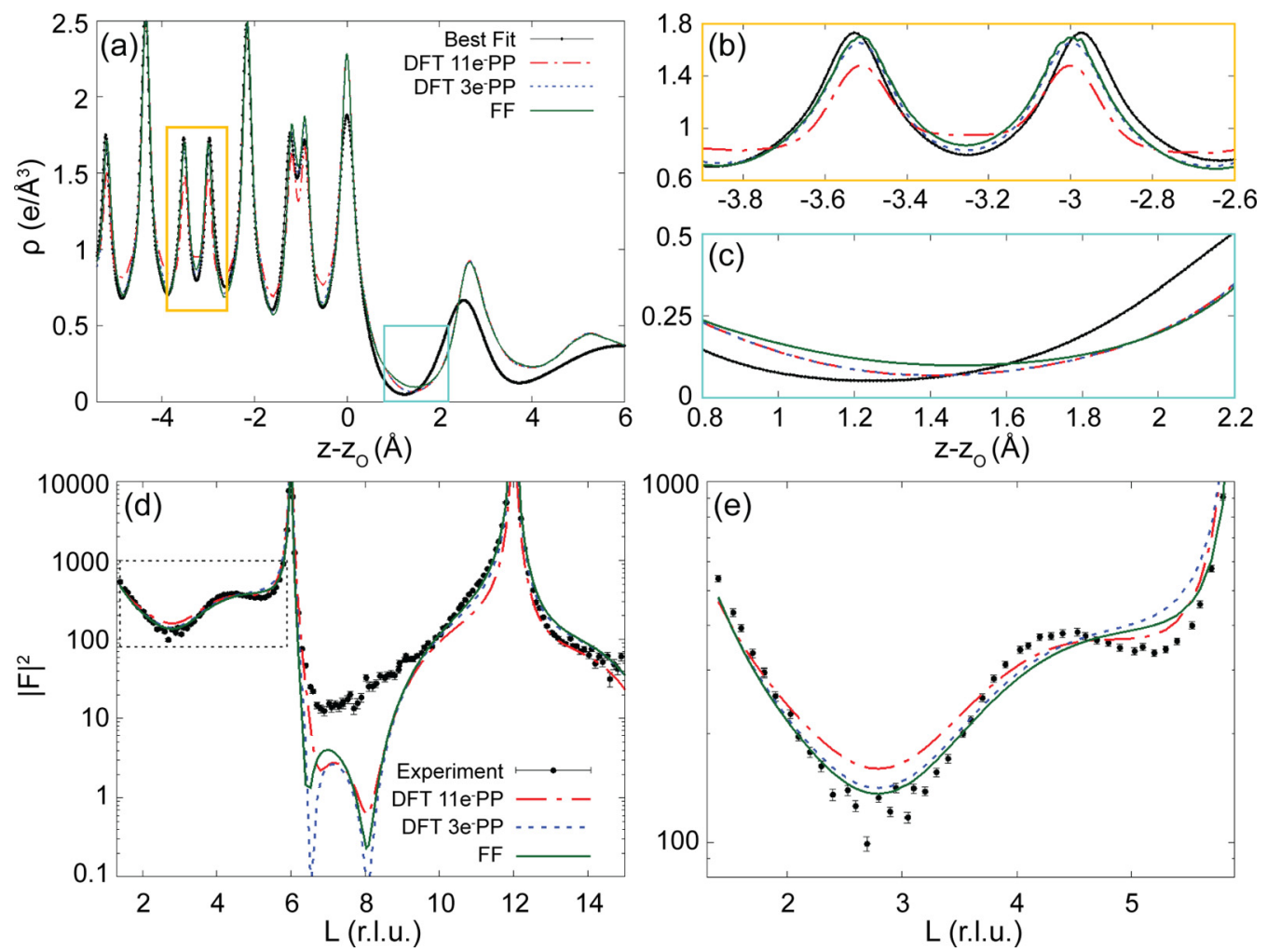

FIG. 7. Effect of different electron-density configurations on the XR signal demonstrated using the atomic density prediction from the optB88 simulation with $10 \%$ compression and $11 e^{-}$PP. (a) The full electron-density profiles $\rho(z)$ calculated directly from the electron-density predictions of the $11 e^{-} \mathrm{PP}$ (red), using the $3 e^{-} \mathrm{PP}$ electron distribution (blue), or using atomic FFs (green) are compared to the experimental best fit (black) with all profiles aligned at the alumina surface oxygen position $z_{0}$. The simulated structures show detailed variations in (b) the interlayer region of the alumina slab (gold box) and in (c) the bonding region at the interface with water (teal box). (b) In the alumina slab, the $11 e^{-} \mathrm{PP}$ electron distribution exhibits an excess density between atomic layers and a decreased $\mathrm{Al}$ peak density. (c) In the bonding region, the electron densities directly from DFT are similar to each other (the $3 e^{-} \mathrm{PP}$ and $11 e^{-} \mathrm{PP}$ cases overlap) and are in closer agreement with the experimental best fit than the density calculated with atomic FFs. (d) The XR intensity $|F|^{2}$ as a function of reciprocal lattice unit $L$ (r.l.u.) computed with the $3 e^{-}$PP agrees best with the XR data (black; experimental errors shown as $1 \sigma$ uncertainties) while the intensity computed directly from the $11 e^{-}$PP electron-density distribution is the least accurate (see Table I). (e) Detailed view of the variation in the low- $L$ region of the computed XR intensities [dotted box in (d)].

densities largely originates from the use of the PP derived with $2 s 2 p$ electrons in the valence partition. Furthermore, the $11 e^{-}$PP XR simulation $\left(\chi^{2}=133\right)$ shows an intensity below the second Bragg peak $(10<L<12)$ that is lower than that seen either for the atomic FF $\left(\chi^{2}=69\right)$ or $3 e^{-} \mathrm{PP}\left(\chi^{2}=57\right)$ results, or in the XR data [Fig. 7(d)], which is indicative of errors in the bulk structure. Hence, we conclude that inaccuracies of the $11 e^{-} \mathrm{PP}$ for $\mathrm{Al}$ contribute to the observed differences in $\chi^{2}$ values (Table I). Also note the significant differences between the $3 e^{-} \mathrm{PP}$ and $11 e^{-} \mathrm{PP} \mathrm{XR}$ intensities below the first Bragg peak [Fig. 7(e)], highlighting that the low- $L$ signal contains information not only about the water structure as we showed earlier, but also about the substrate.

The simulated electron-density distributions in the region between the solid surface and interfacial water [the "bonding region"; Fig. 7(c)] display good qualitative agreement with the experimental density. However, the distribution calculated using atomic FFs shows a higher density in this region than those using DFT charge densities, and it is in slightly worse agreement with the experimental best fit. This likely contributes to the lower accuracy obtained when using atomic FF rather than the $3 e^{-} \operatorname{PP}\left(\chi^{2}=69\right.$ versus 57 , respectively). This higher charge density appears to be associated with the use of atomic FF for neutral atoms $(\mathrm{O}, \mathrm{H})$, which does not account for the possibility of charge transfer at the interface. To test this idea, we also computed the electron-density distributions and $\mathrm{XR}$ intensity from the same atomic density distribution but using ionic FFs $\left(\mathrm{O}^{2-}, \mathrm{H}^{+}\right.$, and $\left.\mathrm{Al}^{3+}\right)$. The use of ionic FFs leads to an electron-density distribution in closer qualitative agreement with that predicted by optB88-10\% DFT charge densities and, consequently, in closer agreement with the experimental best-fit density (Fig. S4 [46]); it also gives a more accurate XR signal with respect to experiment $\left(\chi^{2}=45\right.$; Table SII [46]). These results indicate that the XR data are sensitive to the nature of the charge distribution between $\mathrm{Al}$ and $\mathrm{O}$ at the interface and illustrate the importance of an accurate calculation of electron-density distributions for comparison with experimental XR data so as to avoid the need for any ad hoc choices of form factors.

\section{Role of trajectory sampling}

As a final step of the validation protocol, we analyzed how the statistical sampling of FPMD trajectories may af- 

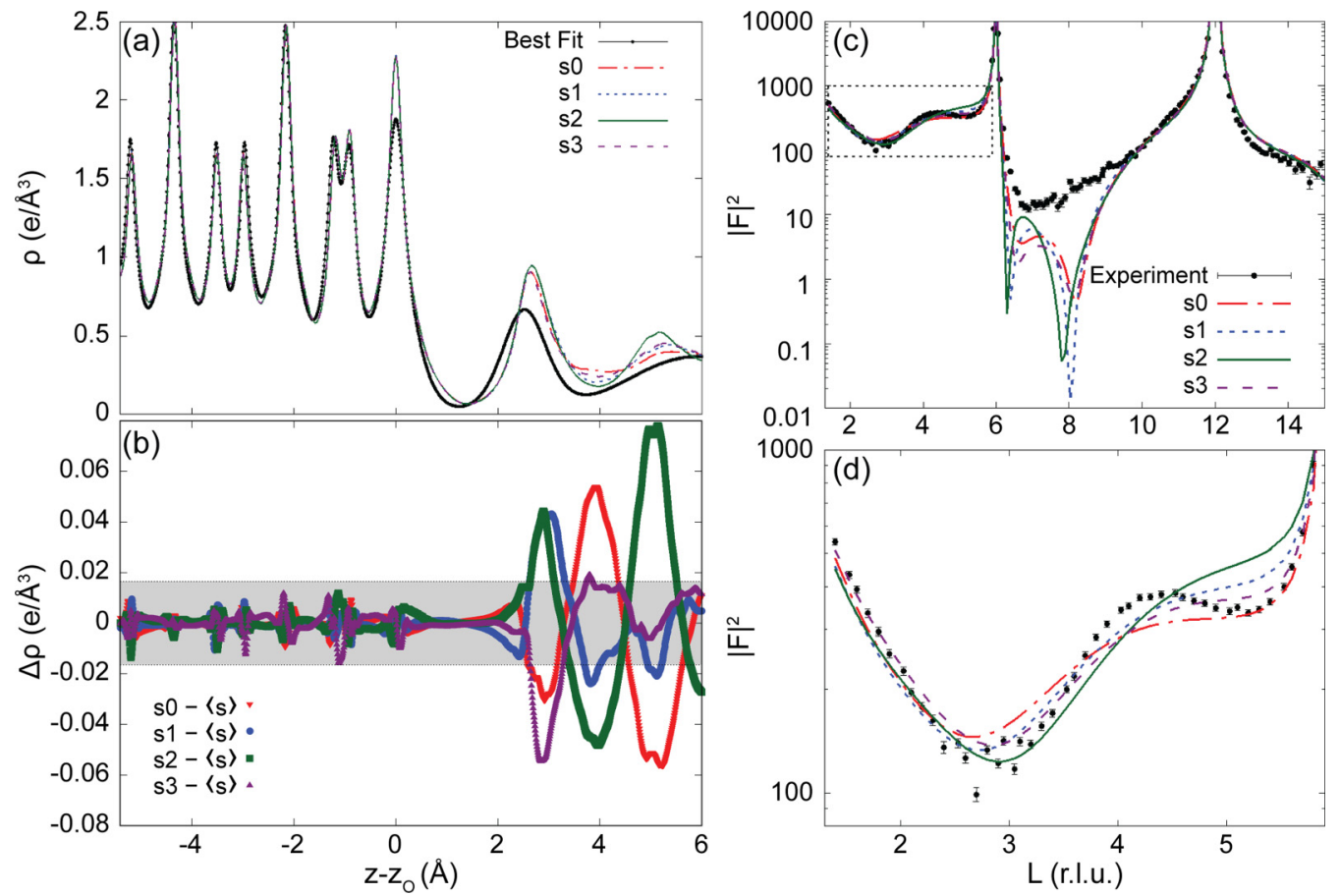

FIG. 8. Role of sampling from four independent trajectories, $s 0-s 3$ ( $s 0$ red; $s 1$ : blue; $s 2$ : green; $s 3$ : purple), using the optB88 simulation with $10 \%$ compression and the $3 e^{-}$PP electron distribution. (a) The trajectories all have similar electron-density distributions $\rho(z)$, but with clear variations in the interfacial water region, $z-z_{\mathrm{O}}>0$ where $z_{\mathrm{O}}$ is the alumina surface oxygen layer position. (b) The difference in density, $\Delta \rho$, between each trajectory and the average of all four trajectories, $\langle s\rangle$ (which gives $\chi^{2}=57$ ), is largest in the water region with the $s 2$ trajectory (green squares) showing the largest deviation; density differences for $s 0-\langle s\rangle$ are shown as red inverted triangles, for $s 1-\langle s\rangle$ as blue circles, and for $s 3-\langle s\rangle$ as purple triangles. The total variance for all trajectories averages zero with a standard deviation of $0.0154 e^{-} / \AA^{3}$ (shaded box). (c) The XR intensities $|F|^{2}$ vs reciprocal lattice unit $L$ (r.l.u.) vary both in the low intensity region $(6<L<8)$ and in (d) the region below the first Bragg peak [dotted box in (c)]. $\chi^{2}$ ranges from $41(s 0)$ to $92(s 2)$. The experimental result is shown for reference (black; experimental errors shown as $1 \sigma$ uncertainties).

fect the accuracy of computed XR signals. We focus on four independent trajectories obtained for the compressed optB88 simulations using the $3 e^{-}$PP (yielding an average accuracy of $\chi^{2}=57$ ). The four trajectories were each sampled for 3.4 ps and show similar electron-density predictions [Fig. 8(a)] with differences appearing primarily in the interfacial water distribution [Fig. 8(b)]. The levels of agreement with the XR data range from $41<\chi^{2}<92$ with two to three order-ofmagnitude differences in the computed XR intensities in the low-intensity region [6 $<L<8$; Fig. 8(c)] and up to a factor of $\sim 2$ differences in the region below the first Bragg peak [Fig. 8(d)]. For completeness, we performed an equivalent analysis for the same trajectories but with atomic FF (Fig. S5 [46]) and for four independent PBE trajectories (Fig. S6 [46]) and found similar results. From these observations, we conclude that the interfacial water region is the main contributor to the variability in $\chi^{2}$ for each FPMD methodology, and it is an important factor in the strong variation of the computed XR intensities at $L=6-8$ across the different simulations.

These results show how the presence of sharp minima in $\mathrm{XR}$ intensities are intrinsically challenging to reproduce by DFT. Specifically, even subtle differences in the electrondensity distributions from trajectory to trajectory (for any given DFT methodology) can lead to significant differences in the computed XR signals that influence the calculated $\chi^{2}$ level of accuracy. Therefore, the robust sampling of multiple trajectories, as previously suggested for pure water [13], is crucial to accurately describe these low-intensity features in the simulated XR signals. We propose that the high sensitivity of these minima in the XR data can, in principle, be used as a stringent test of FPMD-predicted charge densities at oxide/water interfaces, such as those that are controlled by hydrogen bonding.

\section{E. Empirical fitting to XR data}

We showed that the XR data are sensitive to both the solid surface and the water region; hence, they cannot be used to uniquely determine the accuracy of the theoretical predictions of interfacial water (i.e., the quality of agreement in the theory-experiment comparisons is also determined by the accuracy of the substrate structure). However, isolating the contribution of the interfacial water can be achieved by performing model-dependent optimization, guided by the XR data, using the DFT simulations as the starting point. In particular, we preferentially test the accuracy of the interfacial water structure by using the (fixed) as-simulated interfacial water profile but allowing the geometry of the $\mathrm{Al}_{2} \mathrm{O}_{3}$ surface structure to differ from that predicted from FPMD. This is achieved by choosing structural parameters for modeldependent fitting that accurately describe the simulated $\mathrm{Al}_{2} \mathrm{O}_{3}$ interfacial structure (i.e., atom positions and widths described in Secs. II A and II B; see details in Ref. [21] and the Supplemental Material [46]) as starting points, but then we allow 
TABLE II. $\chi^{2}$ fitting results using atomic FFs or charge densities from DFT to describe the electron-density distributions for the three water structures simulated using PBE [38] with the $3 e^{-} \mathrm{PP}$, optB88 [39] with the $11 e^{-} \mathrm{PP}$ and no compression, and optB88 with the $11 e^{-} \mathrm{PP}$ and compression of the unit cell. The $\mathrm{Al}_{2} \mathrm{O}_{3}$ surface atomic structures were relaxed from the as-predicted FPMD simulations in order to separately assess the $\chi^{2}$ contributions relating to the water structure. $\Delta V$ is the volume change of the simulation cell via compression along alumina (001) direction relative to the volume used in the PBE simulation. $\chi_{0}^{2}$ is calculated from the parametrized $\mathrm{Al}_{2} \mathrm{O}_{3}$ surface (see the Supplemental Material and Fig. S7 for details [46]) before the alumina surface structure was optimized; $\chi_{f A}^{2}$ and $\chi_{f D F T}^{2}$ indicate the final quality of agreement of the $\mathrm{Al}_{2} \mathrm{O}_{3}$ /water structures with the experimental data when the electron density of the simulated water is calculated either using atomic FFs or directly from the DFT electronic structure prediction, respectively.

\begin{tabular}{lccccr}
\hline \hline & & & \multicolumn{2}{c}{ Atomic FF } & DFT $e^{-}$ \\
\cline { 3 - 5 } Exchange correlation & PP & $\Delta V(\%)$ & Initial $\left(\chi_{0}^{2}\right)^{\mathrm{a}}$ & Optimized $\left(\chi_{\mathrm{fA}}^{2}\right)$ & 11.2 \\
PBE & 3 & 0 & 124 & 17.0 & 5.6 \\
optB88 & 11 & 0 & 90 & 5.6 & 5.5 \\
\hline
\end{tabular}

${ }^{a}$ These values differ from those calculated directly from the simulations (Table I) due to the use here of the experimental bulk structure rather than the structure derived from the FPMD slab, confirming the need for high accuracy in the simulated bulk structure. Nevertheless, the trends in $\chi^{2}$ are similar, i.e., optB88-10\% has the highest accuracy).

those parameters to change in the optimization. We also use the experimentally known lattice parameter, $d_{006}=2.1663 \AA$, and internal coordinates of alumina [26]. In this optimization procedure, the alumina atom positions and widths change from their initial values (i.e., those simulated by FPMD) to reduce the $\chi^{2}$ error associated with the solid surface, leading to an optimized structure with a $\chi^{2}$ result that is sensitive primarily to errors in the simulated water structures.

The optimized $\chi^{2}$ values are compared in Table II and are substantially smaller than those obtained previously through direct comparisons (Table I). This indicates that inaccuracies of the DFT-simulated atomic displacements in the alumina surface were a major contribution to the large $\chi^{2}$ values. We note that the associated changes in the alumina surface structure upon optimization are small in absolute magnitude, with a mean difference in the atom heights within the top two surface unit cells of only $\sim 0.06 \AA$ (averaged over the PBE, optB88, and optB88-10\% simulations, using both the FFs and the DFT electron densities). The large reduction in $\chi^{2}$ upon geometry optimization reveals that these differences in the alumina surface structure are significant and required to make quantitative comparisons between the measured and simulated XR data. These smaller $\chi^{2}$ values $\left(\chi^{2} \sim 5\right)$ indicate that there is near-quantitative agreement between the simulated hydration structure and the XR data (i.e., $\chi^{2} \approx 1$ ). This approach was then used to assess the relative accuracy of the interfacial water structures from our FPMD simulations using PBE and optB88 with and without compression of the unit cell. Here, we see that the optB88-10\% simulation gives the most accurate description of the interfacial water structure : $\chi_{f A}^{2}=5.6$ and $\chi_{\mathrm{DFT}}^{2}=5.5$ using the water elemental density profile with atomic FF or using the DFT charge density, respectively. The optimized structure using the PBE water as predicted by DFT charge densities results in an equivalent accuracy $\left(\chi_{f D F T}^{2}=\right.$ 5.6), but the fit using the PBE water structure described by atomic FF is less accurate $\left(\chi_{f A}^{2}=11.2\right)$. These results indicate that while a reduced water height and increased water coverage are important to achieve agreement with the XR results, other features of the optimized $\mathrm{Al}_{2} \mathrm{O}_{3}$ /water structures can negatively impact the overall agreement with the XR data.
While the origin of the $\chi^{2}$ trends is not immediately obvious from the interfacial electron-density distributions [Fig. 9(a)], the resulting XR signals [Fig. 9(b)] show better agreement with the XR data for optB88-10\% versus PBE or the larger optB88 simulation as well as for the DFT-based calculations versus those using atomic FFs. We highlight, in particular, the remaining discrepancies with the XR data in the region between the Bragg peaks $(6<L<9)$, which we previously identified as being associated with, but not exclusively due to, the interfacial water. Here, we find that a smaller $\chi^{2}$ is correlated with a larger optimized rms width of the $\mathrm{Al}_{2} \mathrm{O}_{3}$ surface oxygen [Fig. 9(c)]. A broader peak in this layer leads to a slightly larger electron density in the bonding region between the solid and water, which then appears to affect the computed XR intensities and is consistent with the observation of Sec. III D that even subtle changes in the electron density can lead to significant differences in the low-intensity regions of the XR data. This result may indicate sensitivity of the XR data to subtle changes to the interfacial electron density arising from charge transfer or proton distributions at oxide/water interfaces.

\section{CONCLUSIONS}

Following the general protocol outlined in Fig. 1, we have systematically tested theoretical and numerical approximations made in first-principles simulations of the $\mathrm{Al}_{2} \mathrm{O}_{3}$ /water interface, and we have assessed how they affect the accuracy of the predicted structures. Approximations include the choice of exchange correlation functional, pseudopotential, size of the system, and the approach to calculate the experimental observable. We have used $\mathrm{x}$-ray reflectivity as the experimental observable for our validation due to its high sensitivity to both bulk and interfacial structures. We have found, crucially, that XR exhibits extreme sensitivity to electron densities as well as to atomic coordinates. For example, the delocalization of electrons around atomic cores in the bulk substrate, originating from the explicit treatment of semicore electrons, was shown to contribute significantly to inaccuracies in computed XR signals. We also demonstrated an enhanced sensitivity of 

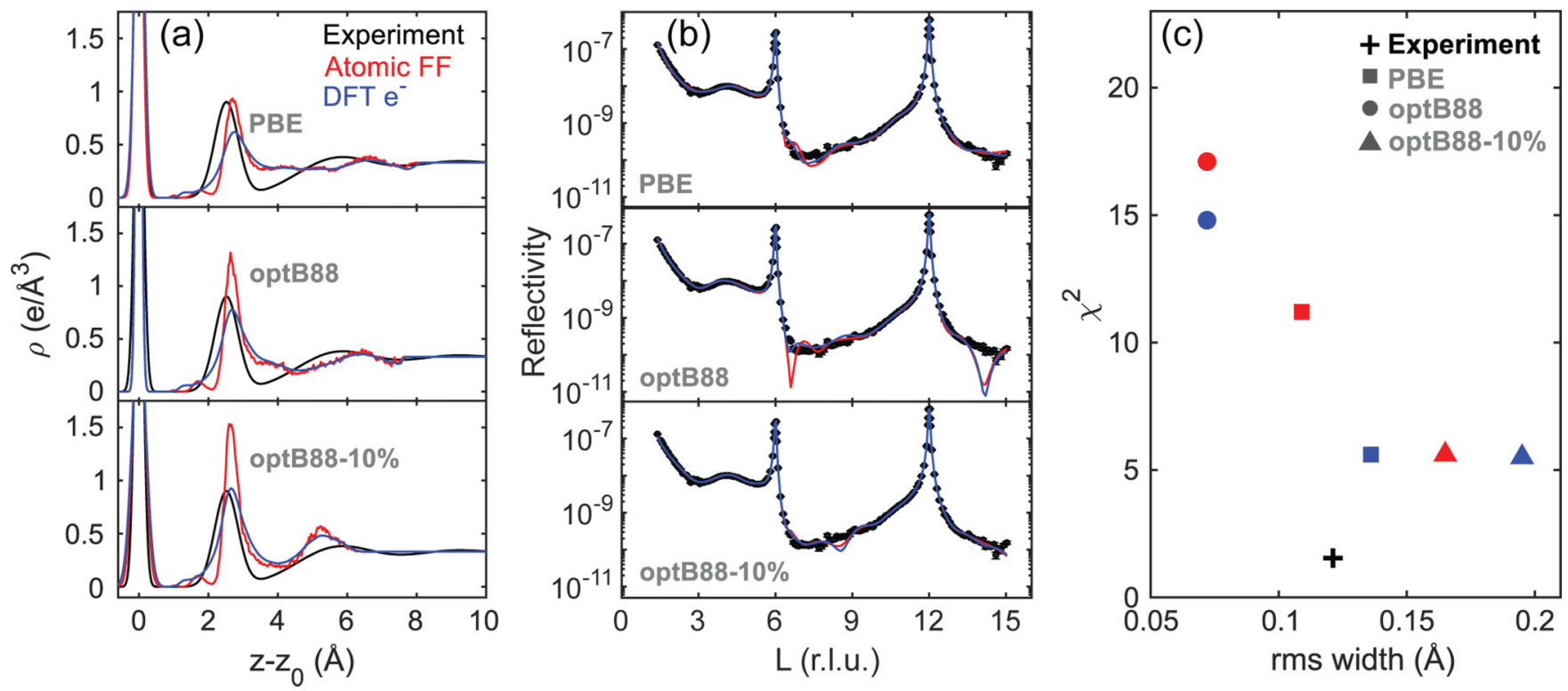

FIG. 9. Optimized alumina interface structures with simulated first-principles molecular dynamics (FPMD) water structures. (a) The electron densities $\rho(z)$ referenced to the position of the alumina surface oxygen at $z_{0}$ from PBE (top), optB88 (middle), and optB88 with $10 \%$ compression, optB88-10\% (bottom) are each compared to the experimental best-fit structure (black line). (b) The final simulated XR intensities $|F|^{2}$ vs reciprocal lattice unit $L$ (r.l.u.) after optimizing the $\mathrm{Al}_{2} \mathrm{O}_{3}$ surface (see Table II for $\chi^{2}$ results; experimental errors shown as $1 \sigma$ uncertainties). (c) The $\chi^{2}$ associated with the structures in (a) are correlated with the optimized rms width of the alumina surface oxygen (PBE results shown as squares, optB88 as circles, and optB88-10\% as triangles). Red data indicate that the water structure was calculated from FPMD atomic number density prediction with atomic form factors; blue data indicate that the water structure was calculated directly from the FPMD electronic structure predictions; black data indicate the experimental result.

low-intensity regions in the XR data to details of the interfacial water distribution and established that the XR data are sensitive to the charge transfer occurring at the solid/water interface. Finally, through a model-dependent optimization of the $\mathrm{Al}_{2} \mathrm{O}_{3}$ surface structure, we were able to isolate inaccuracies associated with the simulated oxide substrate structure and quantify the relative accuracy of FP predictions of interfacial water.

Although none of the simulations evaluated in this work were able to achieve full quantitative agreement when directly compared with the XR data, the comparisons between simulation and experiments presented here provide insights as to what improvements are needed from FP calculations to do so. The present results demonstrate that a fully quantitative and direct evaluation of the interface accuracy of FPMD simulations using XR data relies on having accurate predictions of the bulk substrate and bulk water structures because XR data probe the bulk and interfacial regions simultaneously. We expect that larger simulation cells are needed to eliminate errors in the bulk phases. We estimate that more than $12 \mathrm{Al}_{2} \mathrm{O}_{3}$ layers may be necessary [21] to address this technical challenge. We also conclude that a larger simulation volume is needed to represent the extent of oscillations in the interfacial water region given that the experimental best fit shows the interfacial water density oscillations decaying to bulk at $>10 \AA$ from the alumina surface. Based on our NPT-NVT CMD tests of systems with up to 2520 water molecules (Fig. S1 and Table SI [46]), we expect that a simulation with at least 280 water molecules may be needed to provide a more accurate prediction of the interfacial water behavior than those carried out here with 96 water molecules. However, such large simulation cells are currently beyond the scope of what can be simulated with FPMD in a reasonable amount of time on existing high-performance computers.

While we have focused on the validation of the PBE and optB88 functionals, there are many other flavors of DFT that may be good candidates for the validation protocol described in this study. For example, hybrid functionals [40,49-51] can perform better than semilocal functionals for the structural and diffusion properties of bulk water $[15,52]$ and for some bulk solid phases [53] including $\mathrm{Al}_{2} \mathrm{O}_{3}$ [54]. However, most hybrid functionals cannot accurately describe interfaces between systems with different dielectric properties such as alumina and water. In addition, it is currently unclear how to properly describe $\mathrm{vdW}$ interactions $[55,56]$ within the context of hybrid functionals. The strongly constrained and appropriately normed (SCAN) functional [57] is more computationally advantageous than hybrid functionals and can accurately capture many water properties $[4,58]$. SCAN was recently shown [59] to outperform a hybrid functional (HSE) $[49,50]$ for the prediction of vibrational properties at the $\mathrm{Al}_{2} \mathrm{O}_{3}(001) /$ water interface and the prediction of the adsorbed water height above the alumina surface $(2.62 \AA)$. However, the results presented in Ref. [59] derive from simulations with fewer $\mathrm{Al}_{2} \mathrm{O}_{3}$ layers and fewer water molecules than we used here, which would be insufficient to accurately predict the XR intensities. Nevertheless, the available results point to SCAN as a promising functional for predictions of oxide/water interfaces for future study using this validation framework. 
Finally, the effects of surface protonation should be incorporated explicitly. In this work we assumed a fully hydroxylated alumina surface, consistent with previous simulations [42,59] and experimental results [29-33] at neutral $p \mathrm{H}$. However, it is known that the alumina surface protonation is controlled by $p \mathrm{H}$ (characterized by a point of zero charge) $[33,60,61]$ and that this behavior can be influenced by surface morphology and defects [62-64]. The experimental $\mathrm{Al}_{2} \mathrm{O}_{3}$ sample to which we have compared in this work was cleaned and annealed to eliminate surface defects, and thus the assumption of a fully protonated surface is expected to be reasonable. Still, the interfacial protonation state is known to influence the interfacial structure [65], and consequently, a thorough comparison between the computational and experimental results necessitates that these $p \mathrm{H}$-dependent effects be accounted for in the simulations.

In summary, we have established a framework to test the accuracy of FPMD at interfaces and have identified the breadth of information that can be gleaned through comparisons with XR data. The procedure that we have described can be applied to understand the strengths and weaknesses of various FPMD design choices and ultimately to quantify the accuracy of future simulations as new functionals and computational approaches are developed that enable increasingly scalable calculations [66]. Finally, the technical requirements that are needed for evaluating the ability of FPMD to accurately simulate, and therefore understand, the interactions and structures at the oxide/water interface via a direct 1:1 comparison with XR data have been defined.

The Department of Energy will provide public access to these results of federally sponsored research in accordance with the DOE Public Access Plan [67].

\section{ACKNOWLEDGMENTS}

This work was supported, in part, by the Midwest Integrated Center for Computational Materials (MICCoM) as part of the Computational Materials Sciences Program funded by the U.S. Department of Energy, Office of Science, Basic Energy Sciences (DOE/BES), Materials Sciences and Engineering Division (Grant No. 5J-30161-0010A). This work made use of the Center for Nanoscale Materials (CNM), the Argonne Leadership Computing Facility (ALCF), and beamline 33-ID-D of the Advanced Photon Source at Argonne National Lab (ANL), Office of Science User Facilities supported by the DOE/BES. This material is based upon work supported by Laboratory Directed Research and Development (LDRD) funding from ANL. K.J.H. gratefully acknowledges support from the U.S. Department of Defense through the National Defense Science and Engineering Graduate Fellowship (NDSEG) Program and from the Ryan Fellowship at Northwestern University International Institute of Nanotechnology. K.L.-W. acknowledges the Thomas F. and Kate Miller Jeffress Memorial Trust, Bank of America, Trustee. A.P.G. was supported by the postdoctoral fellowship from the Natural Sciences and Engineering Research Council of Canada. The submitted manuscript has been created by UChicago Argonne, LLC, Operator of Argonne National Laboratory ("Argonne"). Argonne, a U.S. Department of Energy Office of Science laboratory, is operated under Contract No. DEAC02-06CH11357. The U.S. Government retains for itself, and others acting on its behalf, a paid-up nonexclusive, irrevocable worldwide license in said article to reproduce, prepare derivative works, distribute copies to the public, and perform publicly and display publicly, by or on behalf of the Government.
[1] Y. Morikawa, K. Iwata, J. Nakamura, T. Fujitani, and K. Terakura, Ab initio study of surface structural changes during methanol synthesis over $\mathrm{Zn} / \mathrm{Cu}(111)$, Chem. Phys. Lett. 304, 91 (1999).

[2] M. Gerosa, F. Gygi, M. Govoni, and G. Galli, The role of defects and excess surface charges at finite temperature for optimizing oxide photoabsorbers, Nat. Mater. 17, 1122 (2018).

[3] M. Behrens, F. Studt, I. Kasatkin, S. Kühl, M. Hävecker, F. Abild-Pedersen, S. Zander, F. Girgsdies, P. Kurr, B.-L. Kniep et al., The active site of methanol synthesis over $\mathrm{Cu} / \mathrm{ZnO} / \mathrm{Al}_{2} \mathrm{O}_{3}$ industrial catalysts, Science 336, 893 (2012).

[4] M. Chen, H.-Y. Ko, R. C. Remsing, M. F. Calegari Andrade, B. Santra, Z. Sun, A. Selloni, R. Car, M. L. Klein, J. P. Perdew et al., Ab initio theory and modeling of water, Proc. Natl. Acad. Sci. USA 114, 10846 (2017).

[5] A. Alkauskas, B. B. Buckley, D. D. Awschalom, and C. G. Van de Walle, First-principles theory of the luminescence lineshape for the triplet transition in diamond NV centres, New J. Phys. 16, 073026 (2014).

[6] J. Davidsson, V. Ivády, R. Armiento, N. T. Son, A. Gali, and I. A. Abrikosov, First principles predictions of magneto-optical data for semiconductor point defect identification: the case of divacancy defects in 4H-SiC, New J. Phys. 20, 023035 (2018).
[7] F. Ren, L. Ward, T. Williams, K. J. Laws, C. Wolverton, J. Hattrick-Simpers, and A. Mehta, Accelerated discovery of metallic glasses through iteration of machine learning and highthroughput experiments, Sci. Adv. 4, eaaq1566 (2018).

[8] G. I. Csonka, J. P. Perdew, A. Ruzsinszky, P. H. T. Philipsen, S. Lebègue, J. Paier, O. A. Vydrov, and J. G. Ángyán, Assessing the performance of recent density functionals for bulk solids, Phys. Rev. B 79, 155107 (2009).

[9] C. Zhang, J. Wu, G. Galli, and F. Gygi, Structural and vibrational properties of liquid water from van der waals density functionals, J. Chem. Theory Comput. 7, 3054 (2011).

[10] K. Berland and P. Hyldgaard, Exchange functional that tests the robustness of the plasmon description of the van der waals density functional, Phys. Rev. B 89, 035412 (2014).

[11] P. Pernot, B. Civalleri, D. Presti, and A. Savin, Prediction uncertainty of density functional approximations for properties of crystals with cubic symmetry, J. Phys. Chem. A 119, 5288 (2015).

[12] F. Muttaqien, Y. Hamamoto, I. Hamada, K. Inagaki, Y. Shiozawa, K. Mukai, T. Koitaya, S. Yoshimoto, J. Yoshinobu, and Y. Morikawa, $\mathrm{CO}_{2}$ adsorption on the copper surfaces: van der Waals density functional and TPD studies, J. Chem. Phys. 147, 094702 (2017). 
[13] W. Dawson and F. Gygi, Equilibration and analysis of firstprinciples molecular dynamics simulations of water, J. Chem. Phys. 148, 124501 (2018).

[14] K. Lejaeghere, G. Bihlmayer, T. Björkman, P. Blaha, S. Blügel, V. Blum, D. Caliste, I. E. Castelli, S. J. Clark, A. Dal Corso et al., Reproducibility in density functional theory calculations of solids, Science 351, aad3000 (2016).

[15] M. J. Gillan, D. Alfè, and A. Michaelides, Perspective: How good is DFT for water? J. Chem. Phys. 144, 130901 (2016).

[16] P. Haas, F. Tran, and P. Blaha, Calculation of the lattice constant of solids with semilocal functionals, Phys. Rev. B 79, 085104 (2009).

[17] F. Tran, J. Stelzl, and P. Blaha, Rungs 1 to 4 of DFT Jacob's ladder: Extensive test on the lattice constant, bulk modulus, and cohesive energy of solids, J. Chem. Phys. 144, 204120 (2016).

[18] P. Fenter, S. Kerisit, P. Raiteri, and J. D. Gale, Is the calcitewater interface understood? direct comparisons of molecular dynamics simulations with specular $\mathrm{x}$-ray reflectivity data, J. Phys. Chem. C 117, 5028 (2013).

[19] J. N. Bracco, S. S. Lee, J. E. Stubbs, P. J. Eng, F. Heberling, P. Fenter, and A. G. Stack, Hydration structure of the barite (001)water interface: comparison of $\mathrm{x}$-ray reflectivity with molecular dynamics simulations, J. Phys. Chem. C 121, 12236 (2017).

[20] S. J. T. Brugman, P. Raiteri, P. Accordini, F. Megens, J. D. Gale, and E. Vlieg, Calcite (104) surface-electrolyte structure: a $3 \mathrm{~d}$ comparison of surface $\mathrm{x}$-ray diffraction and simulations, J. Phys. Chem. C 124, 18564 (2020).

[21] K. J. Harmon, Y. Chen, E. J. Bylaska, J. G. Catalano, M. J. Bedzyk, J. H. Weare, and P. Fenter, Insights on the aluminawater interface structure by direct comparison of density functional simulations with x-ray reflectivity, J. Phys. Chem. C 122, 26934 (2018).

[22] M. Plaza, X. Huang, J. Y. Ko, M. Shen, B. H. Simpson, J. Rodriguez-Lopez, N. L. Ritzert, K. Letchworth-Weaver, D. Gunceler, D. G. Schlom et al., Structure of the photocatalytically active surface of $\mathrm{SrTiO}_{3}$, J. Am. Chem. Soc. 138, 7816 (2016).

[23] P. Fenter and S. S. Lee, Hydration layer structure at solid-water interfaces, MRS Bull. 39, 1056 (2014).

[24] P. A. Fenter, X-ray reflectivity as a probe of mineral-fluid interfaces: a user guide, Rev. Mineral. Geochem. 49, 149 (2002).

[25] C. J. Yu, A. G. Richter, A. Datta, M. K. Durbin, and P. Dutta, Molecular layering in a liquid on a solid substrate: an X-ray reflectivity study, Phys. B (Amsterdam, Neth.) 283, 27 (2000).

[26] A. Kirfel and K. Eichhorn, Accurate structure analysis with synchrotron radiation. The electron density in $\mathrm{Al}_{2} \mathrm{O}_{3}$ and $\mathrm{Cu}_{2} \mathrm{O}$, Acta Crystallogr., Sect. A 46, 271 (1990).

[27] I. Robinson, X-ray crystallography of surfaces and interfaces, Acta Crystallogr., Sect. A 54, 772 (1998).

[28] P. J. Brown, A. G. Fox, E. N. Maslen, M. A. O'Keefe, and B. T. M. Willis, in International Tables for Crystallography, edited by E. Prince (Springer, Dordrecht, 2006), Vol. C, Chap. 6.1, p. 554.

[29] J. G. Catalano, Weak interfacial water ordering on isostructural hematite and corundum (001) surfaces, Geochim. Cosmochim. Acta 75, 2062 (2011).

[30] J. W. Elam, C. E. Nelson, M. A. Cameron, M. A. Tolbert, and S. M. George, Adsorption of $\mathrm{H}_{2} \mathrm{O}$ on a single-crystal $\alpha-\mathrm{Al}_{2} \mathrm{O}_{3}(0001)$ surface, J. Phys. Chem. B 102, 7008 (1998).
[31] P. Liu, T. Kendelewicz, G. E. Brown, E. J. Nelson, and S. A. Chambers, Reaction of water vapor with $\alpha-\mathrm{Al}_{2} \mathrm{O}_{3}(0001)$ and $\alpha-\mathrm{Fe}_{2} \mathrm{O}_{3}(0001)$ surfaces: synchrotron X-ray photoemission studies and thermodynamic calculations, Surf. Sci. 417, 53 (1998).

[32] P. J. Eng, T. P. Trainor, G. E. Brown Jr., G. A. Waychunas, M. Newville, S. R. Sutton, and M. L. Rivers, Structure of the hydrated $\alpha-\mathrm{Al}_{2} \mathrm{O}_{3}$ (0001) surface, Science 288, 1029 (2000).

[33] L. Zhang, C. Tian, G. A. Waychunas, and Y. R. Shen, Structures and charging of $\alpha$-alumina (0001)/water interfaces studied by sum-frequency vibrational spectroscopy, J. Am. Chem. Soc. 130, 7686 (2008).

[34] K. C. Hass, W. F. Schneider, A. Curioni, and W. Andreoni, Firstprinciples molecular dynamics simulations of $\mathrm{H}_{2} \mathrm{O}$ on $\alpha-\mathrm{Al}_{2} \mathrm{O}_{3}$ (0001), J. Phys. Chem. B 104, 5527 (2000).

[35] F. Gygi, Architecture of Qbox: A scalable first-principles molecular dynamics code, IBM J. Res. Dev. 52, 137 (2008), accessible at http://qboxcode.org.

[36] D. R. Hamann, Optimized norm-conserving vanderbilt pseudopotentials, Phys. Rev. B 88, 085117 (2013).

[37] M. Schlipf and F. Gygi, Optimization algorithm for the generation of ONCV pseudopotentials, Comput. Phys. Commun. 196, 36 (2015).

[38] J. P. Perdew, K. Burke, and M. Ernzerhof, Generalized Gradient Approximation Made Simple, Phys. Rev. Lett. 77, 3865 (1996).

[39] J. Klimeš, D. R. Bowler, and A. Michaelides, Chemical accuracy for the van der waals density functional, J. Phys.: Condens. Matter 22, 022201 (2010).

[40] C. Adamo and V. Barone, Toward reliable density functional methods without adjustable parameters: the PBE0 model, J. Chem. Phys. 110, 6158 (1999).

[41] C. Zhang, D. Donadio, F. Gygi, and G. Galli, First principles simulations of the infrared spectrum of liquid water using hybrid density functionals, J. Chem. Theory Comput. 7, 1443 (2011).

[42] P. Huang, T. A. Pham, G. Galli, and E. Schwegler, Alumina(0001)/water interface: structural properties and infrared spectra from first-principles molecular dynamics simulations, J. Phys. Chem. C 118, 8944 (2014).

[43] S. Plimpton, Fast parallel algorithms for short-range molecular dynamics, J. Comput. Phys. 117, 1 (1995).

[44] R. T. Cygan, J.-J. Liang, and A. G. Kalinichev, Molecular models of hydroxide, oxyhydroxide, and clay phases and the development of a general force field, J. Phys. Chem. B 108, 1255 (2004).

[45] H. J. C. Berendsen, J. R. Grigera, and T. P. Straatsma, The missing term in effective pair potentials, J. Phys. Chem. 91, 6269 (1987).

[46] See Supplemental Material at http://link.aps.org/supplemental/ 10.1103/PhysRevMaterials.4.113805 for further simulation details, CMD results, comparisons with PBE0 and 11e- PP and with ionic FFs, additional statistical sampling results, and details of the empirical fitting procedure.

[47] K. W. Corum, X. Huang, J. W. Bennett, and S. E. Mason, Systematic density functional theory study of the structural and electronic properties of constrained and fully relaxed $\left(\begin{array}{lll}0 & 0 & 1\end{array}\right)$ surfaces of alumina and hematite, Mol. Simul. 43, 406 (2017).

[48] F. Herbstein, How precise are measurements of unit-cell dimensions from single crystals? Acta Crystallogr., Sect. B 56, 547 (2000). 
[49] J. Heyd, G. E. Scuseria, and M. Ernzerhof, Hybrid functionals based on a screened Coulomb potential, J. Chem. Phys. 118, 8207 (2003).

[50] J. Heyd, G. E. Scuseria, and M. Ernzerhof, Erratum: "Hybrid functionals based on a screened coulomb potential" [J. Chem. Phys. 118, 8207 (2003)], J. Chem. Phys. 124, 219906 (2006).

[51] A. V. Krukau, O. A. Vydrov, A. F. Izmaylov, and G. E. Scuseria, Influence of the exchange screening parameter on the performance of screened hybrid functionals, J. Chem. Phys. 125, 224106 (2006).

[52] A. P. Gaiduk, J. Gustafson, F. Gygi, and G. Galli, Firstprinciples simulations of liquid water using a dielectricdependent hybrid functional, J. Phys. Chem. Lett. 9, 3068 (2018).

[53] H. Zheng, M. Govoni, and G. Galli, Dielectric-dependent hybrid functionals for heterogeneous materials, Phys. Rev. Mater. 3, 073803 (2019).

[54] J. H. Skone, M. Govoni, and G. Galli, Self-consistent hybrid functional for condensed systems, Phys. Rev. B 89, 195112 (2014).

[55] R. A. DiStasio, Jr., B. Santra, Z. Li, X. Wu, and R. Car, The individual and collective effects of exact exchange and dispersion interactions on the ab initio structure of liquid water, J. Chem. Phys. 141, 084502 (2014).

[56] F. Ambrosio, G. Miceli, and A. Pasquarello, Structural, dynamical, and electronic properties of liquid water: A hybrid functional study, J. Phys. Chem. B 120, 7456 (2016).

[57] J. Sun, A. Ruzsinszky, and J. P. Perdew, Strongly Constrained and Appropriately Normed Semilocal Density Functional, Phys. Rev. Lett. 115, 036402 (2015).

[58] M. D. LaCount and F. Gygi, Ensemble first-principles molecular dynamics simulations of water using the SCAN meta-GGA density functional, J. Chem. Phys. 151, 164101 (2019).
[59] M. J. DelloStritto, S. M. Piontek, M. L. Klein, and E. Borguet, Effect of functional and electron correlation on the structure and spectroscopy of the $\mathrm{Al}_{2} \mathrm{O}_{3}(001)-\mathrm{H}_{2} \mathrm{O}$ interface, J. Phys. Chem. Lett. 10, 2031 (2019).

[60] J. P. Fitts, X. Shang, G. W. Flynn, T. F. Heinz, and K. B. Eisenthal, Electrostatic surface charge at aqueous $/ \alpha-\mathrm{Al}_{2} \mathrm{O}_{3}$ single-crystal interfaces as probed by optical second-harmonic generation, J. Phys. Chem. B 109, 7981 (2005).

[61] A. G. Stack, S. R. Higgins, and C. M. Eggleston, Point of zero charge of a corundum-water interface probed with optical second harmonic generation (SHG) and atomic force microscopy (AFM): New approaches to oxide surface charge, Geochim. Cosmochim. Acta 65, 3055 (2001).

[62] B. Braunschweig, S. Eissner, and W. Daum, Molecular structure of a mineral/water interface: effects of surface nanoroughness of $\alpha-\mathrm{Al}_{2} \mathrm{O}_{3}$ (0001), J. Phys. Chem. C 112, 1751 (2008).

[63] M. Kosmulski, The pH-dependent surface charging and the points of zero charge, J. Colloid Interface Sci. 253, 77 (2002).

[64] G. V. Franks and L. Meagher, The isoelectric points of sapphire crystals and alpha-alumina powder, Colloids Surf., A 214, 99 (2003).

[65] Z. Zhang, P. Fenter, N. C. Sturchio, M. J. Bedzyk, M. L. Machesky, and D. J. Wesolowski, Structure of rutile $\mathrm{TiO}_{2}$ (110) in water and $1 \mathrm{molal} \mathrm{Rb}+$ at $\mathrm{pH} 12$ : Inter-relationship among surface charge, interfacial hydration structure, and substrate structural displacements, Surf. Sci. 601, 1129 (2007).

[66] L. Zhang, J. Han, H. Wang, R. Car, and W. E, Deep Potential Molecular Dynamics: A Scalable Model with the Accuracy of Quantum Mechanics, Phys. Rev. Lett. 120, 143001 (2018).

[67] https://www.energy.gov/downloads/doe-public-access-plan. 